\title{
Estacionalidad de la erosión y el transporte eólico de partículas en el desierto costero de Atacama, Chile ( $\left(3^{\circ} \mathrm{S}\right)$
}

\author{
Valentina Flores-Aqueveque ${ }^{1}$, Gabriel Vargas ${ }^{1}$, José Rutllant² ${ }^{2}$ Jacobus P. Le Roux ${ }^{1}$
}

\author{
${ }^{1}$ Departamento de Geología, Facultad de Ciencias Físicas y Matemáticas, Universidad de Chile. Plaza Ercilla 803, Santiago, Chile. \\ v.flores.a@gmail.com; gvargas@ing.uchile.cl;jroux@ing.uchile.cl \\ 2 Departamento de Geofisica, Facultad de Ciencias Físicas y Matemáticas, Universidad de Chile. Blanco Encalada 2002, Santiago, Chile. \\ jrutllan@dgf.uchile.cl
}

\begin{abstract}
RESUMEN. La costa del Desierto de Atacama se caracteriza por la ocurrencia de vientos fuertes del sur y suroeste. El clima árido, la geomorfología plana y las características granulométricas de los depósitos superficiales de la Pampa Mejillones, ubicada en la costa del norte de Chile $\left(23^{\circ} \mathrm{S}\right)$, favorecen el estudio de los procesos de erosión y transporte eólico de partículas en este desierto costero. Mediciones in situ del flujo horizontal de partículas (G) y de vientos, junto con análisis sedimentológicos de la capa superficial de esta pampa, permitieron determinar los parámetros relevantes en el proceso de erosión y transporte eólico de partículas, así como estimar su estacionalidad. Se determinó un coeficiente de rugosidad superficial $\left(\mathrm{z}_{0}\right)$ promedio del orden de $10^{-3} \mathrm{~m}$, típico de planicies desérticas. Estacionalmente se observaron valores de $\mathrm{G}$ de hasta $2.100 \mathrm{~g} \mathrm{~cm}^{-1} \mathrm{mes}^{-1}$ asociados a velocidades de fricción ( $\mathrm{u}^{*}$ ) entre 0,4 y $0,5 \mathrm{~m} \mathrm{~s}^{-1}$ durante la primavera del año 2000. Resultados similares se obtuvieron para los años 1999 y 2001. La sedimentología (granulometría y textura superficial) y mineralogía de las partículas del suelo y del material capturado en las trampas de polvo, permitieron determinar que la fracción transportada está caracterizada principalmente por cuarzo y feldespatos, y modas granulométricas entre 75 y $1.000 \mu \mathrm{m}$, similares a las observadas en los sedimentos cercanos y al sur (viento arriba) del área de captura. Los resultados obtenidos y la correlación preliminar entre los vientos medidos en distintas estaciones meteorológicas en la península de Mejillones, permiten establecer una relación directa entre el flujo horizontal de partículas hacia la bahía homónima y los vientos regionales del sur-suroeste, especialmente durante los meses de primavera y verano, en concomitancia con la intensificación de los vientos favorables a la surgencia costera en el área oceánica adyacente.
\end{abstract}

Palabras claves: Mejillones, Atacama, Erosión, Transporte eólico, Coeficiente de rugosidad superficial, Velocidad de fricción, Surgencia costera.

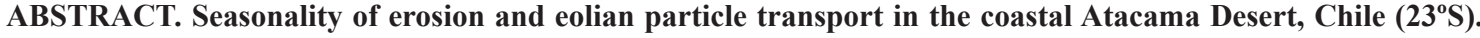
The coast of the Atacama Desert is characterized by the occurrence of strong south- and southwesterly winds. The arid climate, flat geomorphology and the characteristics of the superficial sediments on Pampa Mejillones, located in northern Chile $\left(\mathrm{ca} .23^{\circ} \mathrm{S}\right)$, favor the study of eolian erosion and transport processes in this coastal desert. In situ measurements of the horizontal particle flux $(\mathrm{G})$ and winds in the area, together with sedimentological analyses of the uppermost part of the pampa sediments, allowed determination of the significant parameters in the eolian erosion and particle transport process and its seasonality. A local surface roughness parameter $\left(\mathrm{z}_{0}\right)$ of about $10^{-3} \mathrm{~m}$ was calculated, which is a typical value of desert plains. Seasonally, values of $\mathrm{G}$ up to $2,100 \mathrm{~g} \mathrm{~cm}^{-1} \mathrm{month}^{-1}$ were observed, associated with friction velocities $\left(\mathrm{u}^{*}\right)$ between 0,4 and $0,5 \mathrm{~m} \mathrm{~s}^{-1}$ during the spring of 2000. Similar results were obtained for the years 1999 and 2001. The sedimentology (granulometry and surface texture) and mineralogy of the surface and captured material in the dust traps show that the transported fraction is characterized mainly by quartz and feldspars, with grain-size modes between 75 and $1,000 \mu \mathrm{m}$, similar to those observed in nearby soils and south (upwind) of the capture area. The results obtained and preliminary correlation of measured winds at the different meteorological stations on the Mejillones Peninsula, indicate a direct relationship between the horizontal particle flux towards Mejillones Bay and the regional south-southwest winds, especially during spring and summer, simultaneous with the intensification of winds favoring coastal upwelling in the area.
\end{abstract}

Keywords: Mejillones, Atacama, Eolian particle erosion and transport, Surface roughness coefficient, Friction velocity, Coastal upwelling. 


\section{Introducción}

La erosión y el transporte eólico de partículas constituyen parte de los procesos fundamentales en el modelado de la superficie terrestre. Su estudio es aplicable, por ejemplo, a los estudios de avance de dunas (Nishimori y Tanaka, 2003), desertificación y deflación de suelos agrícolas (Gomes et al., 2003b; Rajot et al., 2003), dispersión de contaminantes (Adetunji et al., 2001), y reconstrucciones paleoclimáticas y paleooceanográficas (Thompson et al., 2000; Poore et al., 2005; Vargas et al., 2004). Estudios de dinámica sedimentaria han sido desarrollados en las regiones áridas y semiáridas del planeta, como en el nordeste de España (Gomes et al., 2003a), el desierto de Arizona (Nickling y Gillies, 1989), el desierto de Sahara (Marticorena y Bergametti, 1996; Rajot et al., 2003; BreuningMadsen y Awadzi, 2005), desierto de Gobi (Wang et al., 2006), Pampa de la Joya en Perú (Bailey, 1906), Nuevo México (Holliday, 1990) y Australia (Hesse y McTainsh, 2003), entre otros. Estas regiones constituyen, además, las principales áreas de emisión de aerosoles terrígenos a la atmósfera. Sin embargo, estudios cuantitativos de los procesos vinculados a la producción y transporte eólico de partículas en el desierto costero de Atacama no han sido realizados.

A lo largo de la costa del norte de Chile, la aridez inducida por el anticiclón subtropical es reforzada por la Cordillera de los Andes (Rutllant et al., 2003), las aguas frías del Sistema de la Corriente de Humboldt (Strub et al., 1998) y fenómenos de surgencia costera forzados por los vientos del sur durante todo el año (Strub et al., 1998; Rutllant et al., 1998) (Fig. 1). La alta frecuencia estival de cielos despejados, sumado a la mayor influencia del anticiclón especialmente en primavera, intensifican estos vientos a lo largo de la costa, particularmente durante las horas de la tarde cuando el gradiente térmico tierra-mar es más intenso (Rutllant et al., 1998). Esta interacción atmósfera-océano-continente es amplificada en puntas, cabos y penínsulas, tales como Punta Angamos en la península de Mejillones $\left(23^{\circ} \mathrm{S}\right)$, a lo largo de los cuales se favorece la surgencia de aguas frías en la costa (Rutllant et al., 1998; Rutllant y Montecino, 2002) (Fig. 1).

La geomorfología de la península de Mejillones está caracterizada por bloques alzados y deprimidos de orientación norte-sur, con una plataforma costera tectónicamente emergida, en la cual se ex- ponen paleocordones litorales pleistocenos (Pampa Mejillones) o terrazas marinas pleistocenas (Punta Angamos) (Fig. 1) (Ortlieb et al., 1996). Las características sedimentológicas de la Pampa Mejillones, una extensa planicie (400 $\mathrm{km}^{2}$ aproximadamente) situada en el sector norte de la península, consiste en grava fina a gruesa de regular madurez textural (Folk, 1951), con abundantes restos de conchas de moluscos y gastrópodos marinos pleistocenos (Ortlieb et al., 1996) y matriz arenosa, no consolidada, cubiertos en parte por una costra salina de poco espesor (1-2 $\mathrm{cm})$. Los depósitos de estos paleocordones litorales están separados por artesas amplias, cubiertas por arena eólica con numerosas óndulas y megaóndulas, cuyo rumbo promedio este-oeste y asimetría hacia el norte evidencian la predominancia del viento del sur en el área (Fig. 2). La variabilidad espacial en las dimensiones de estas estructuras de origen eólico, además, pone de manifiesto la ocurrencia esporádica de episodios de vientos muy intensos desde el sur y suroeste en el área. Esto, junto con la aridez extrema de esta región, interrumpida sólo ocasionalmente por episodios de lluvia intensa asociados a algunos eventos de El Niño (Vargas et al., 2000), favorecen la erosión y el transporte eólico de partículas hacia la bahía de Mejillones.

\section{Teoría del transporte eólico de partículas}

La capacidad del viento de remover sedimento depende fundamentalmente del tamaño, forma y/o densidad de las partículas. Según esto, los mecanismos posibles de transporte son tres: tracción, suspensión y saltación. La saltación realiza entre el $55-72 \%$ del transporte eólico en los desiertos, movilizando partículas de tamaño entre 70-500 $\mu \mathrm{m}$ (Pye, 1987), siendo las inferiores a $100 \mu \mathrm{m}$ las más susceptibles de ser transportadas (Bagnold, 1941; Alfaro, 1997).

La teoría del perfil vertical logarítmico de velocidades del viento en la capa atmosférica superficial, definida como las primeras decenas de metros directamente influenciados por la fricción superficial, permite establecer una relación entre la variación de la velocidad del viento $(u)$ con la altura $(z)$, la velocidad de fricción $\left(u^{*}\right)$ y el parámetro de rugosidad superficial $\left(z_{0}\right)$, válida para condiciones adiabáticas o de estabilidad atmosférica neutra (Fig. 3) (Stull, 1988). En condiciones no muy alejadas de la neutralidad, como aquellas derivadas de un calentamiento solar en el día y de un enfriamiento radiativo nocturno 


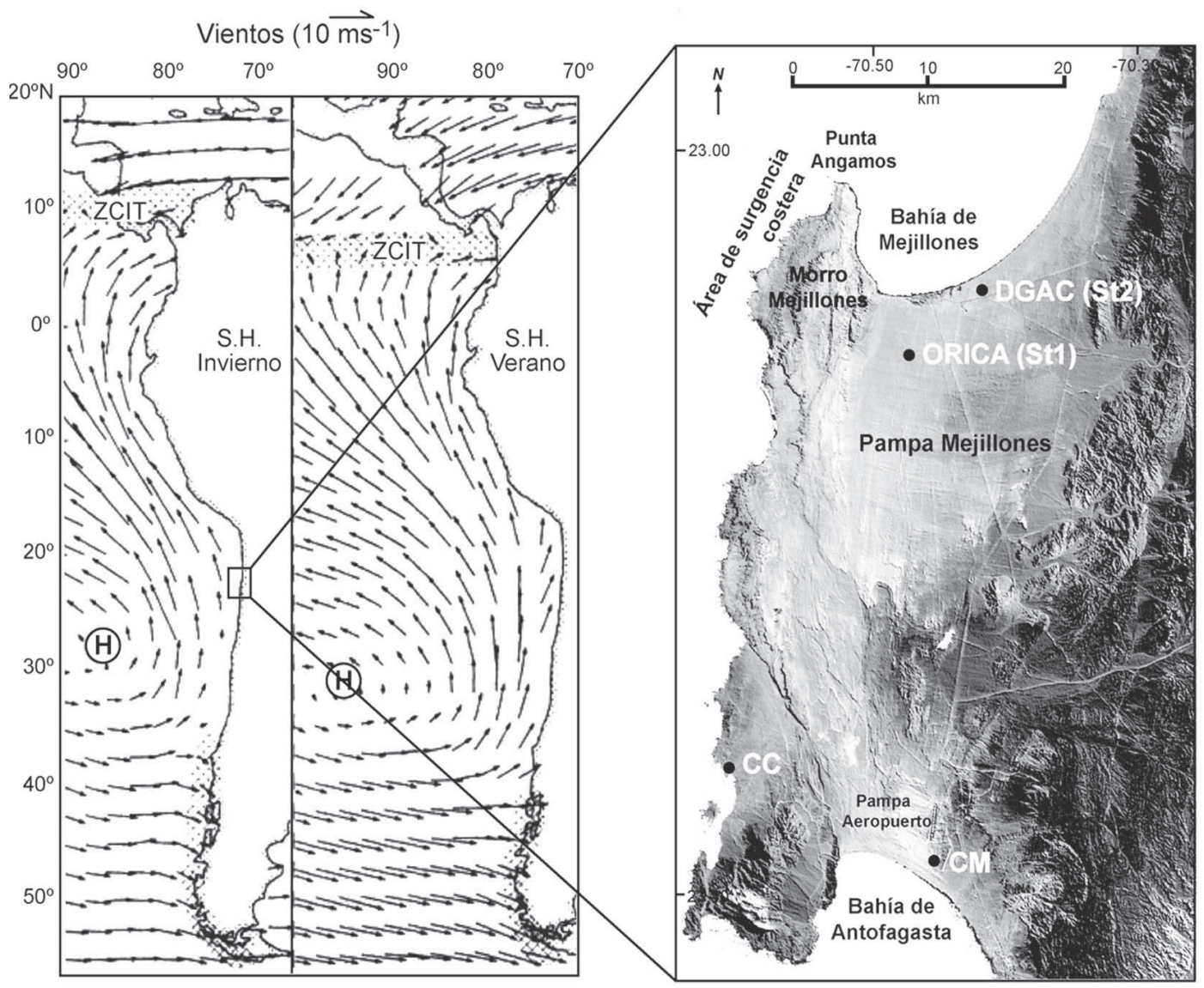

FIG. 1. Ubicación de la península de Mejillones en la costa occidental de América del Sur. Derecha: Estaciones meteorológicas (CC: Caleta Constitución; CM: Cerro Moreno, Orica) y de muestreo de sedimentos (Orica, DGAC) y principales rasgos geomorfológicos. Izquierda: esquema de la circulación superficial del viento en la costa (modificado de Strub et al., 1998).

moderadas por el viento, se utiliza el perfil log-lineal del viento cerca de la superficie para el cálculo de $u^{*}($ Stull, 1988).

La erosión y transporte se inician cuando la velocidad de fricción excede la velocidad de fricción umbral $\left(u_{t}^{*}\right)$ definida como la velocidad mínima para movilizar una partícula de tamaño, forma y densidad dadas. Diversos autores (e.g., Bagnold, 1941; Iversen y White, 1982; Marticorena y Bergametti, 1995; Alfaro, 1997; Le Roux, 1997) han propuesto una relación entre $u^{*}$ y el diámetro de la partícula transportada $D p$ (Fig. 4), indicando que los factores de importancia en el transporte de partículas son la velocidad, la energía turbulenta del viento, y la granulometría del sedimento.

La acción del viento genera dos componentes del flujo de partículas, una horizontal $(G)$ que transporta los granos por saltación y una vertical $(F)$ que los moviliza por suspensión (Marticorena y Bergametti, 1995). Experimentalmente se ha observado que, para valores altos de $u^{*}, G$ es directamente proporcional a $u^{* 3}$ (Bagnold, 1941; Marticorena y Bergametti, 1995). Ambas componentes, $G$ y $F$, están relacionadas de modo tal que la producción de partículas en suspensión, requiere del bombardeo de partículas de mayor tamaño $(60-2.000 \mu \mathrm{m})$, que destruyen los agregados de partículas finas $(<20 \mu \mathrm{m})$ mantenidas en contacto por cohesión, inyectándolas verticalmente a la atmósfera (sandblasting) (Alfaro et al., 2004).

En este trabajo se determinan y cuantifican los parámetros pertinentes para los procesos de transporte eólico horizontal de partículas $(G)$ desde la Pampa Mejillones $\left(23^{\circ} \mathrm{S}\right)$ hacia la bahía homónima, determinando su variabilidad estacional, las condiciones atmosféricas y variaciones climáticas 

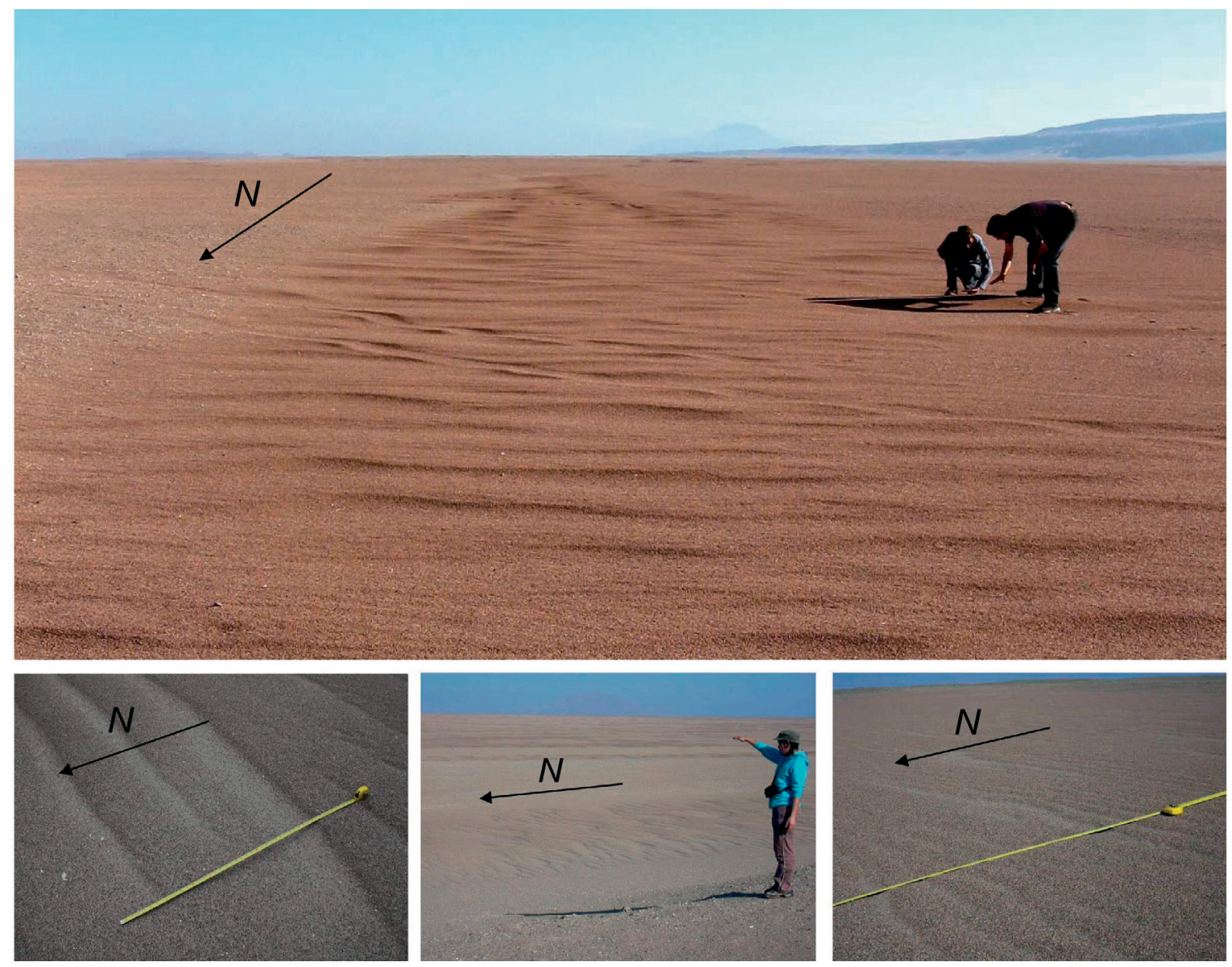

FIG. 2. Estructuras formadas por la acción del viento predominante del sur en los sedimentos de la Pampa Mejillones. Notar distintas escalas de tamaños.

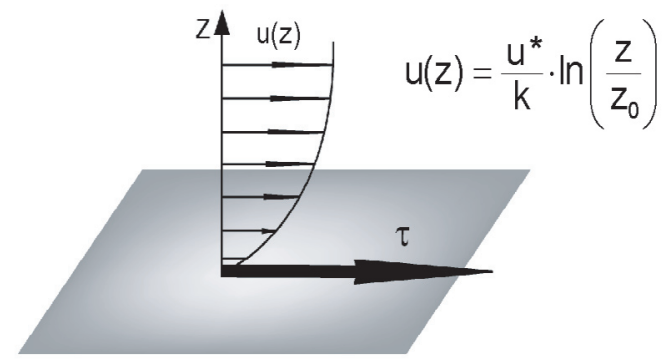

FIG. 3. Ecuación que representa el perfil vertical de velocidades de viento en condiciones de atmósfera neutra cerca de la superficie (Stull, 1988). Notar escala lineal en el eje vertical.

responsables de ésta, su relación con los vientos locales y regionales característicos del área, así como la textura superficial, la granulometría y la mineralogía del material transportado.

\section{Metodología}

\subsection{El experimento EOLOS}

Con el fin de determinar los procesos que intervienen en la erosión y el transporte de partículas en la Pampa Mejillones se desarrolló el experimento EOLOS-2000 entre el 21 de enero y el 8 de febrero de 2000. Durante la realización de este experimento se midió la velocidad media del viento cada 15 minutos a $0,5,1,2$ y $4 \mathrm{~m}$ de altura en la estación Orica (St1, Fig. 1), a partir de lo cual se calcularon los parámetros $u^{*}$ y $z_{0}$. Para el mismo período se analizaron los vientos medidos cada 15 minutos a $4 \mathrm{~m}$ de altura en la estación DGAC (St2, Fig. 1) y los vientos medidos en Caleta Constitución frente a la isla Santa María (CC, Fig. 1), y en el aeropuerto de Cerro Moreno en Antofagasta (CM, Fig. 1). Los datos analizados corresponden a la velocidad y dirección del viento 


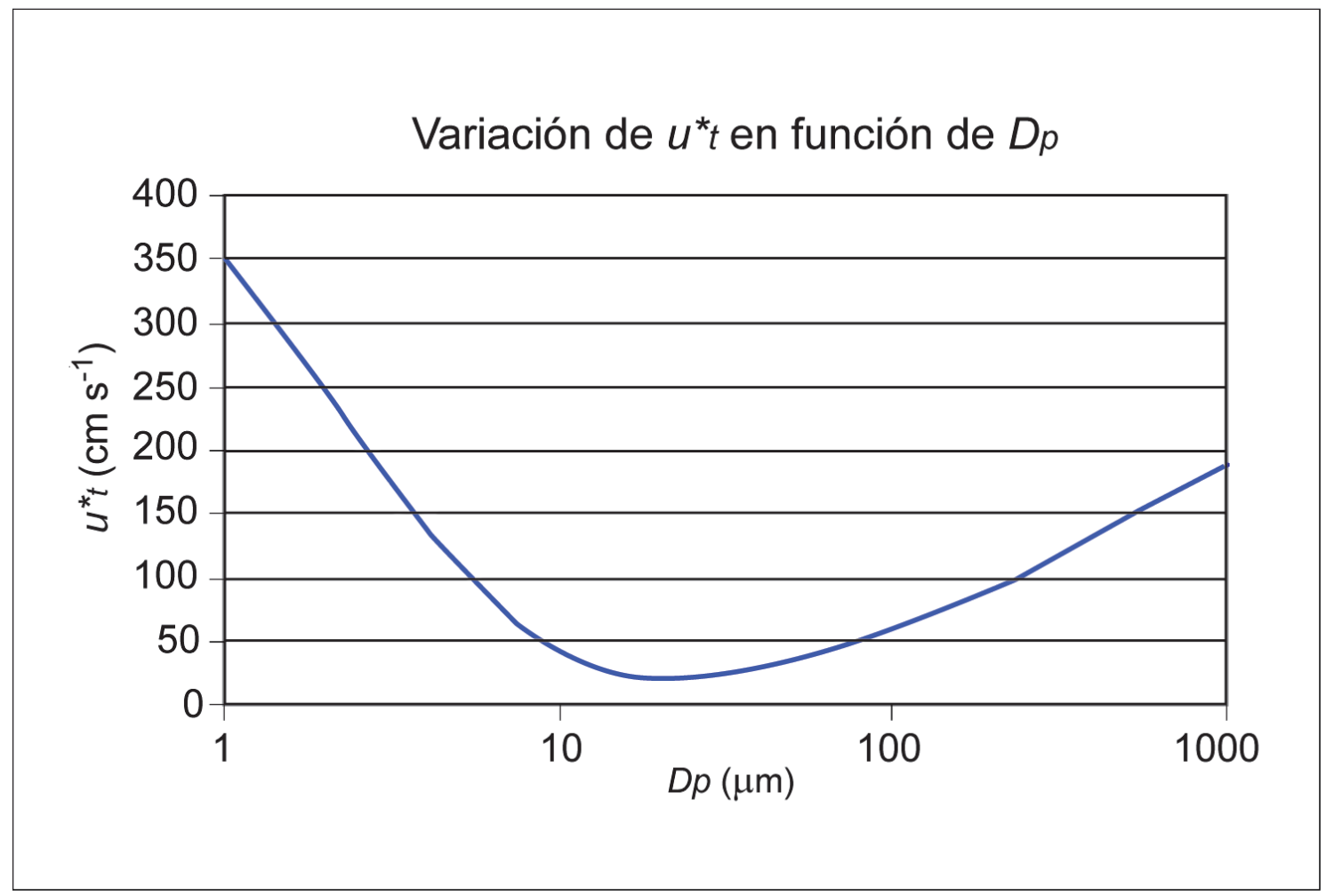

FIG. 4. Relación entre la velocidad de fricción umbral del viento $\left(u^{*}\right)$ y tamaño $(D p)$ de las partículas (consideradas esféricas). Se observa que el $D p_{\text {ópt }}$ que requiere de la menor velocidad para ser transportado se ubica entre 20 y $80 \mu \mathrm{m}$ (modificado de Alfaro, 1997).

medidas a $4 \mathrm{~m}$ sobre la superficie y promediados vectorialmente cada 15 y 30 minutos en la estación Caleta Constitución y DGAC respectivamente, y mediciones puntuales cada 60 minutos a $10 \mathrm{~m}$ de altura en Cerro Moreno. El período analizado en estas dos últimas estaciones comprende los días 20, 21,22 y 23 de enero del año 2000.

El flujo horizontal de partículas $G$ se calculó a través de un monitoreo mensual in situ durante el período 1999-2001, por medio de trampas de captura de partículas instaladas en las estaciones Orica (St1) y DGAC (St2) (Fig. 1). Cada sistema de captura consistió en una torre de $2,5 \mathrm{~m}$ de altura con tres trampas fijas, ubicadas a 10,51 y $112 \mathrm{~cm}$ de altura en Orica (St1), y a 8,50 y $110 \mathrm{~cm}$ de altura en DGAC (St2), orientadas según la dirección del viento predominante del sur (Fig. 5). Las trampas fueron elaboradas en acrílico basadas en el diseño de Fryrear (1986).

Se analizó la granulometría, mineralogía y textura superficial del material capturado durante el año
2000 en Orica (St1) y durante junio, septiembre y octubre de 2000, en DGAC (St2), con el objetivo de determinar sus posibles variaciones temporales y espaciales.

Se realizó un análisis de muestras de sedimentos de distintos sectores de la Pampa Mejillones, con el fin de caracterizar la textura superficial, granulometría y composición de las partículas susceptibles de ser transportadas por el viento. Las muestras recolectadas corresponden a los primeros $0,5 \mathrm{~cm}$ desde la superficie de un área de 50x65 cm. Los sitios de muestreo fueron escogidos considerando la heterogeneidad del terreno, fundamentalmente en función de (1) un chequeo visual de la granulometría de las partículas del sedimento y de las estructuras sedimentarias de origen eólico (óndulas y megaóndulas), (2) la observación preliminar de imágenes satelitales y fotos aéreas de la zona, y (3) su ubicación respecto de las estaciones meteorológicas y de las trampas de captura (Fig. 6). 

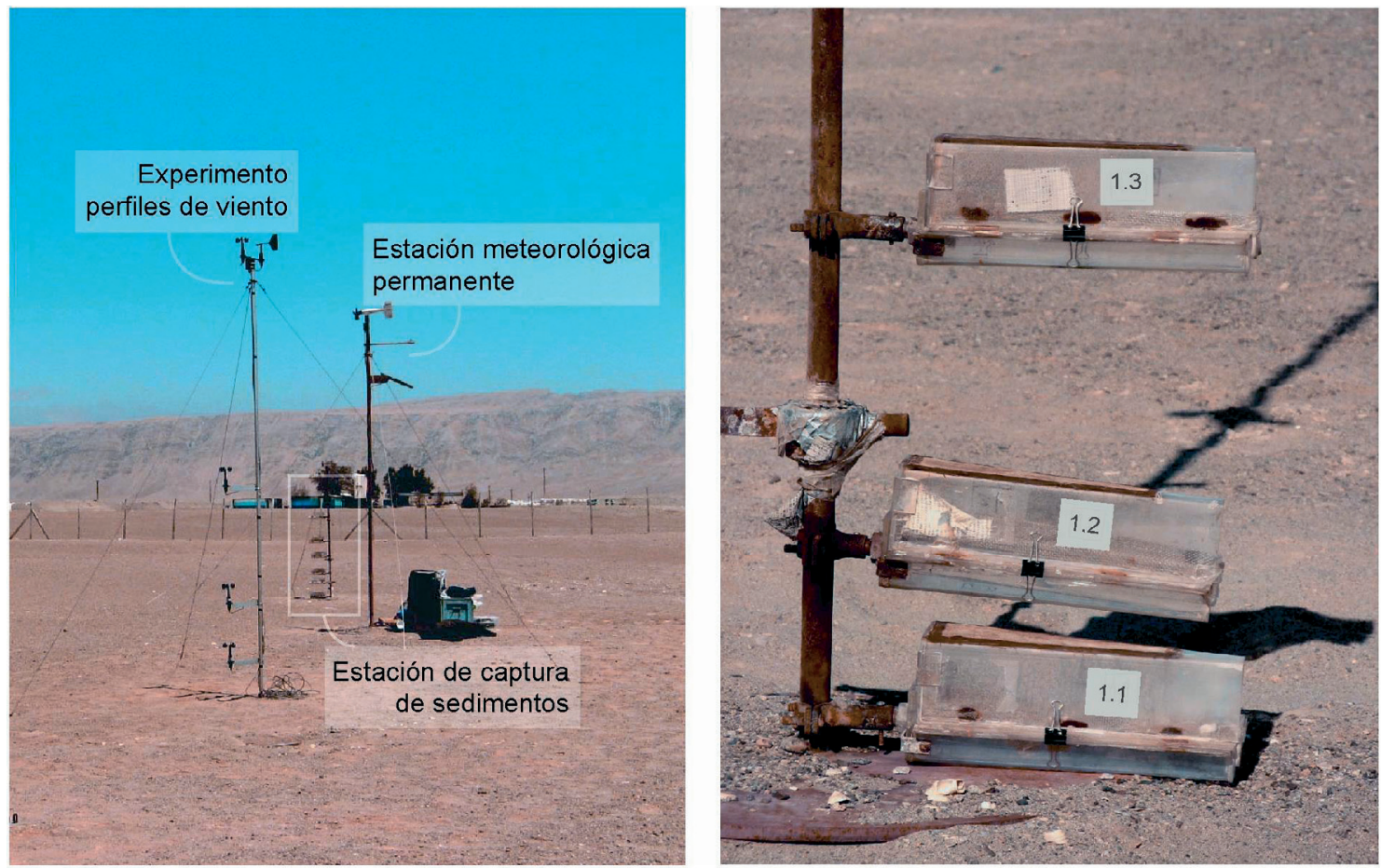

FIG. 5. Estación meteorológica automática permanente instalada en la estación Orica (St1). Mástil para medir el perfil vertical del viento y estación de captura de sedimentos, con el detalle de las trampas BSNE que permanecen fijas hacia el sur (derecha).

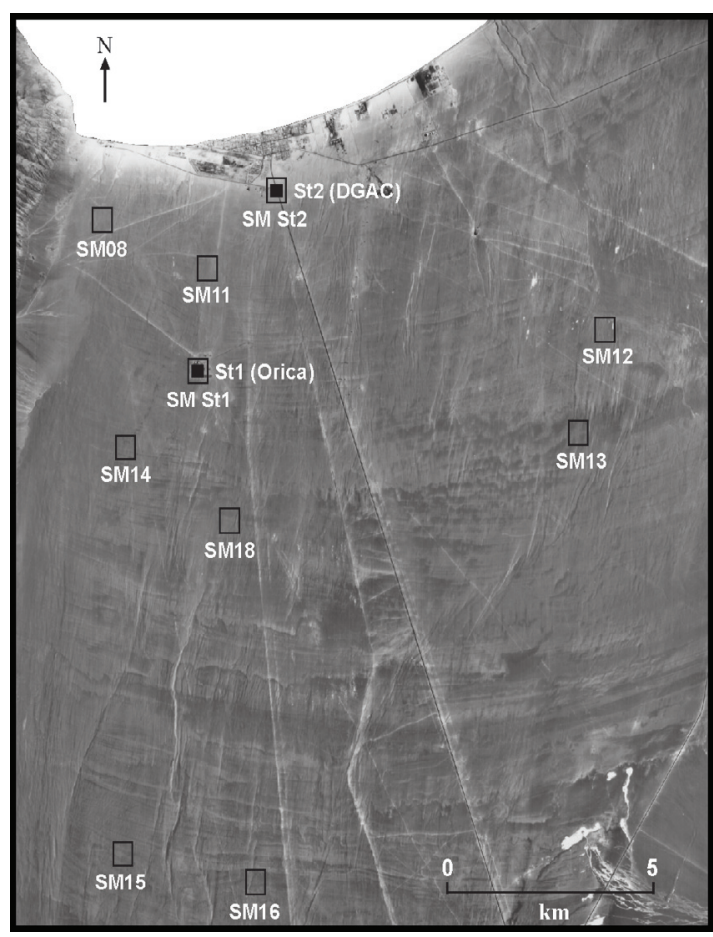

FIG. 6. Ubicación de las muestras de sedimento recolectadas en la Pampa Mejillones.

\subsection{Cálculo de la velocidad de fricción $(u *)$ y el coeficiente de rugosidad $\left(z_{0}\right)$}

Se determinaron los valores de ambas variables sobre la base de los datos de los perfiles verticales de velocidad del viento promediados cada 5 minutos y recopilados entre el 21 de enero y el 8 de febrero de 2000 en la estación Orica (St1). Los datos medidos por el anemómetro instalado a $0,5 \mathrm{~m}$ de altura se marginaron del análisis, ya que la comparación con el resto de los anemómetros mostró diferencias conducentes a error.

Los valores de $u^{*}$ se determinaron optimizando las ecuaciones generales de Businger-Dyer para $u^{*}$ y $q_{0}$, siendo este último el flujo turbulento vertical de calor, positivo para condiciones estables, negativo en condiciones de inestabilidad y nulo en condiciones neutras (Stull, 1988). La altura de rugosidad $\left(z_{0}\right)$ se estimó como el promedio de los $z_{0}$ determinados según la fórmula de Prandtl-von Karman a partir de los perfiles individuales establecidos como neutros, durante viento del sur, sobre la base del perfil logarítmico de velocidades (Stull, 1988) (Figs. 3 y 7).

Con el fin de estimar la variabilidad estacional e interanual de $u^{*}$ en el área para el período 2000- 


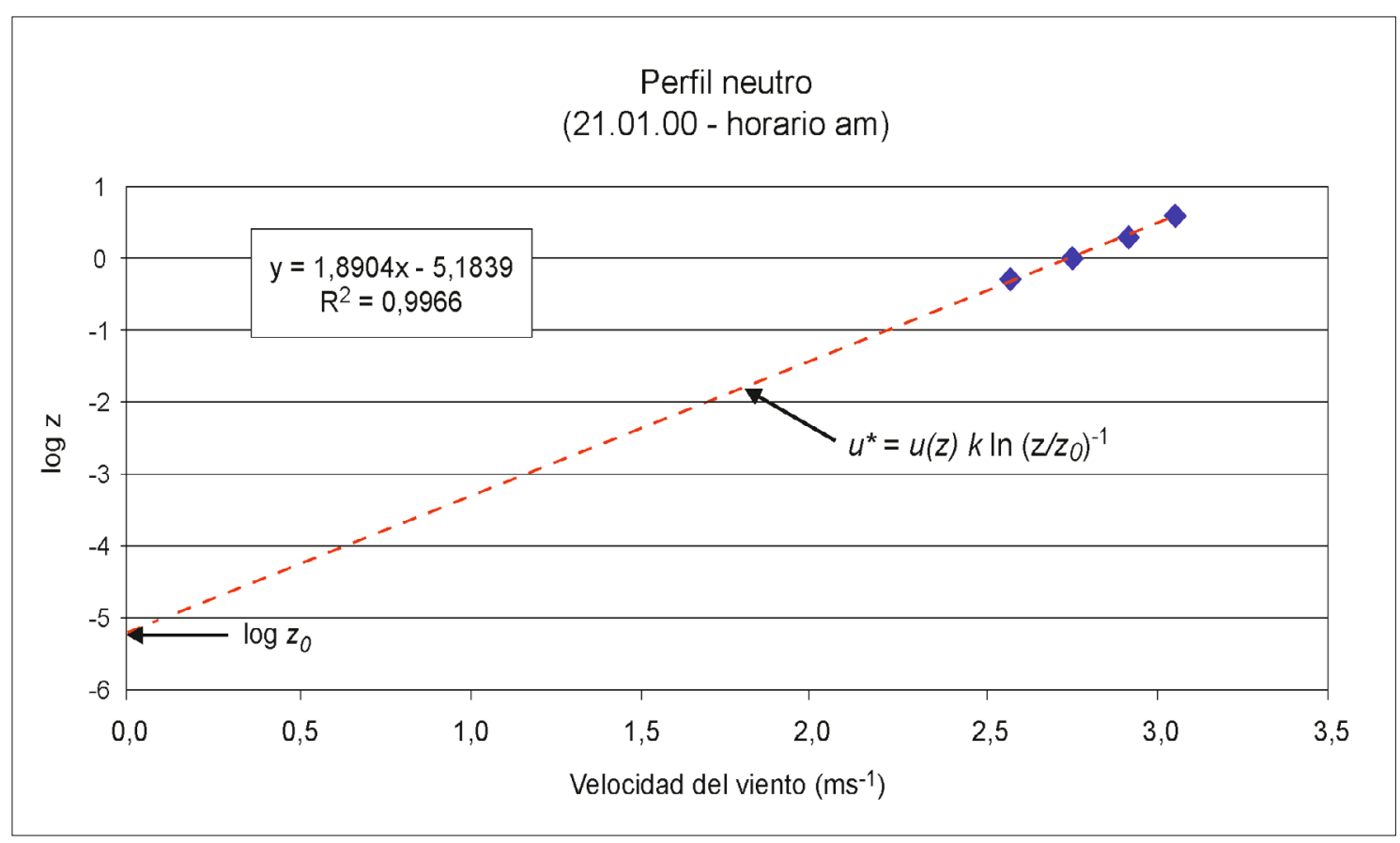

FIG. 7. Ejemplo de la determinación del coeficiente de rugosidad $\left(z_{0}\right)$ y de la velocidad de fricción $\left(u^{*}\right)$ en la estación Orica (St1) a partir del perfil logarítmico de velocidades de viento en condiciones de atmósfera casi neutra para el 21 de enero de 2000.

2005, se calculó en la estación Orica (St1) la componente meridional del seudoesfuerzo superficial del viento $\left(s \tau_{y}\right)$ como el producto entre la magnitud del viento medida a $4 \mathrm{~m}$ de altura $(V(4))$ y su componente meridional (v), es decir, la velocidad del viento en la dirección norte-sur dada por $v=-V \cos (\delta)\left(m s^{-1}\right)$, en que $\delta$ es la dirección del viento en grados a partir del norte $\left(0^{\circ}\right)$.

La correlación local entre los valores de los promedios horarios de $u^{*}$, obtenidos a partir de los perfiles verticales de viento medidos entre el 21 de enero y el 8 de febrero de 2000, con los promedios horarios positivos (viento del sur) de $s \tau_{y}$ calculados para el mismo período en esta estación alcanza un valor de $\mathrm{R}^{2}=0,69$ (Fig. 8). Posteriormente, en consecuencia, se utilizó esta relación para estimar los valores de $u^{*}$ a partir de las mediciones del viento a $4 \mathrm{~m}$ de altura para el período 2000-2005.

\subsection{Flujo horizontal total de partículas $(G)$, medido in situ}

Se calculó un flujo parcial $\left(\mathrm{g}_{\text {parcial }}\right)$ para cada trampa de polvo, considerando la masa del material transportado $m(\mathrm{~g})$, el área de captura $A\left(\mathrm{~cm}^{2}\right)$ y la duración del muestreo $d$ (días) según la fórmula:

$$
g_{\text {parcial }}=C \cdot\left(\frac{m}{A \cdot d}\right)\left(\mathrm{gcm}^{-2}\right)
$$

donde $\mathrm{C}=30$ indica que el flujo se normaliza a un mes de 30 días para la comparación entre períodos.

Dado que el transporte de partículas disminuye exponencialmente con la altura (z) (Stout y Zobeck, 1996) y considerando que el flujo total corresponde a la cantidad (masa) de partículas que pasan por un área de ancho determinado y altura igual al espesor de la atmósfera, el flujo horizontal total de partículas $G$ se calculó a partir de la integración con la altura entre 0 e infinito de la curva exponencial $\left(G_{\text {parcial }}\right)$ determinada por los $g_{\text {parcial }}$ de cada período:

$$
\begin{gathered}
G_{\text {parcial }}=a \exp (-b z) \\
G=\int_{0}^{\infty} a \exp (-b z) d z=-\left.\frac{a}{b} \exp (-b z)\right|_{0} ^{\infty}=-\frac{a}{b}(0-1) \\
G=\frac{a}{b}\left(\mathrm{gcm}^{-1} \mathrm{mes}^{-1}\right)
\end{gathered}
$$

donde $a$ y $b$ son constantes de dimensiones $\mathrm{g} \mathrm{cm}^{-2}$ $\mathrm{mes}^{-1} \mathrm{y} \mathrm{cm}^{-1}$, respectivamente. 


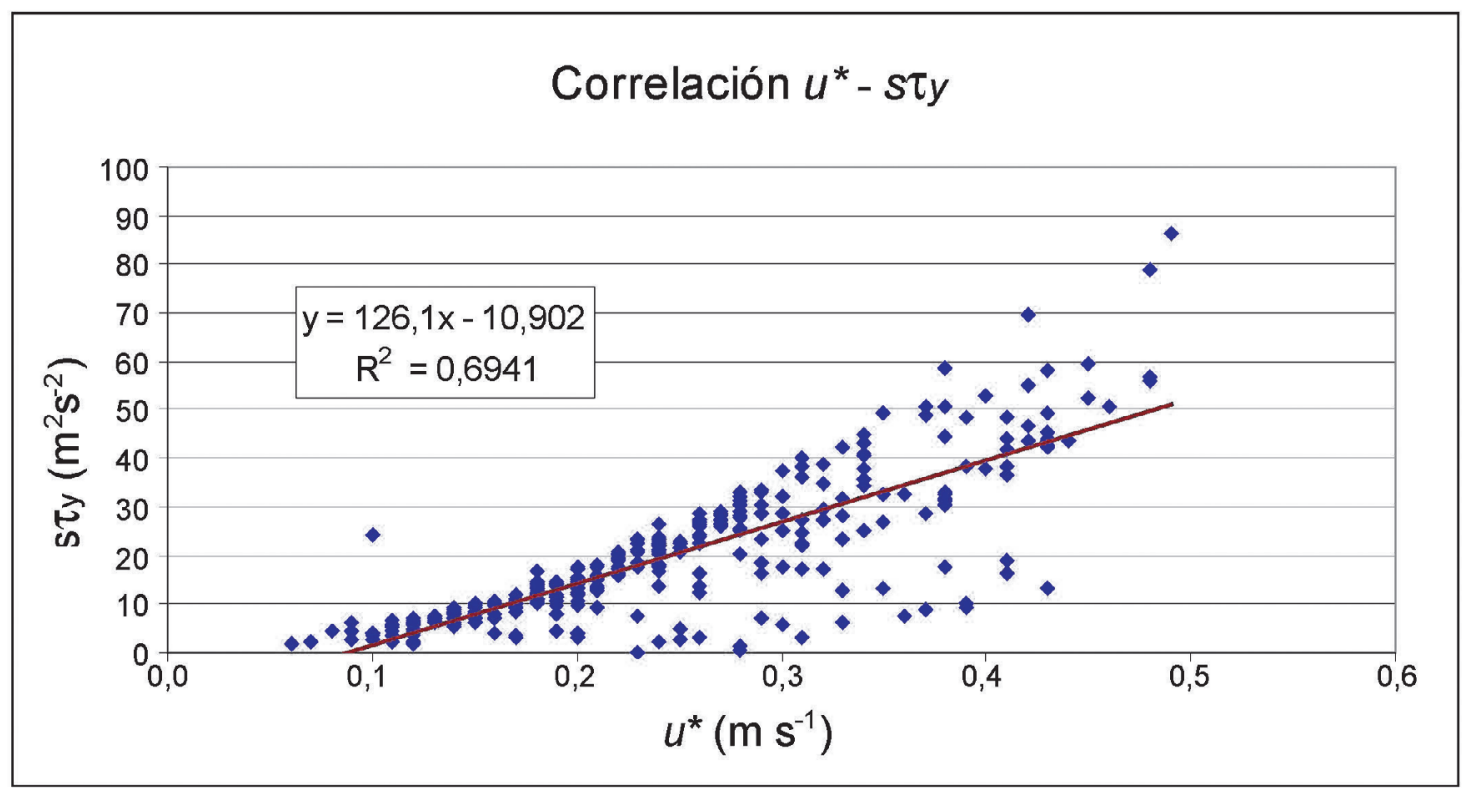

FIG. 8. Correlación lineal entre los promedios horarios de la velocidad de fricción $\left(u^{*}\right)$ y de la componente meridional del seudoesfuerzo superficial del viento $\left(s \tau_{y}\right)$ durante el experimento EOLOS-2000.

El muestreo de las trampas BSNE se realizó para ambas estaciones con regularidad de manera mensual durante todo el año 2000. Adicionalmente, se consideraron las muestras obtenidas durante los años 1999 y 2001 para los cuales el muestreo se realizó en forma irregular. En efecto, los tiempos de muestreo fueron de 69, 46 y 102 días para los períodos marzo-mayo, mayo-julio y julio-septiembre de 1999, y de 81, 143 y 46 días para los períodos marzo-mayo, junio-septiembre y octubre-noviembre de 2001.

\subsection{Características sedimentológicas y minera- lógicas de los sedimentos de la pampa y del material transportado}

\subsubsection{Granulometría y textura superficial}

Se determinó la granulometría de cada muestra de suelo y de las partículas recolectadas en las trampas BSNE para las tres alturas monitoreadas en la estación Orica (St1) (10,51 y $112 \mathrm{~cm}$ de altura sobre la superficie) durante todo el año 2000 por tamizado en seco con el fin de evitar la desagregación de las partículas cohesionadas y obtener una distribución granulométrica de tamaño de grano de las partículas transportadas. La clasificación granulométrica utilizada para este propósito fue la de Udden (1898) modificada por Wentworth (1922).

Se estudió la textura superficial de las partículas del suelo por observación al microscopio electrónico de barrido. Además, se realizaron análisis texturales por medio de lupa binocular a las partículas capturadas a $10 \mathrm{~cm}$ de altura en la estación Orica (St1) durante octubre del 2000, correspondiente al mes con mayor flujo $(G)$ de partículas capturadas de todo el período. Se analizó por el mismo método la textura superficial de las partículas eólicamente transportables $(D p \leq 1.000 \mu \mathrm{m})$, así como los tamaños principales de cada muestra de suelo.

\subsubsection{Mineralogía}

Se estudió la mineralogía, por observación a la lupa binocular, de la fracción eólicamente transportable $(D p \leq 1.000 \mu \mathrm{m})$ del suelo, así como a los intervalos de tamaños más frecuentes de cada muestra. Se realizaron, además, análisis mineralógicos a muestras de suelo por difracción de rayos X.

También se analizó a la lupa binocular la composición del material capturado a $10 \mathrm{~cm}$ de altura en Orica (St1) durante octubre de 2000. 


\section{Resultados}

\subsection{Régimen local de vientos en Orica (St1) y DGAC (St2)}

Mediciones de viento realizadas durante el experimento EOLOS-2000 en Orica (St1) y DGAC (St2) muestran un régimen local de vientos en la Pampa Mejillones (Fig. 9) caracterizado por magnitudes de la componente meridional (v), similares en ambas estaciones, aunque con valores levemente inferiores para la estación DGAC (St2) principalmente durante las horas de la tarde. Los mínimos $\left(\mathrm{v}_{\text {promedio }}<2,0 \mathrm{~m} \mathrm{~s}^{-1}\right)$ se observan en la madrugada y mañana. A partir de las 12:00 hrs aproximadamente se observa un incremento de las velocidades hasta alrededor de las 18:00 hrs donde alcanzan valores máximos $\left(\mathrm{v}_{\text {promedio }} \sim 3,0 \mathrm{~m} \mathrm{~s}^{-1}\right)$.

\subsection{Valores de $z_{0}$ y velocidad de fricción}

Los valores de $z_{0}$ se estimaron a partir de 37 perfiles verticales de velocidad del viento (Fig. 7) durante condiciones casi neutras con vientos del sur. Los resultados varían entre $3,925 \times 10^{-11}$ y $2,72 \times 10^{-1} \mathrm{~m}$, con un promedio de $3,027 \times 10^{-3} \mathrm{~m}$.

Los valores de $u^{*}$ calculados para el mismo período oscilaron entre $0,06-0,49 \mathrm{~m} \mathrm{~s}^{-1}$ (Fig. 10), con un promedio de $0,249 \mathrm{~m} \mathrm{~s}^{-1} \mathrm{y}$ una desviación estándar $\sigma=0,095 \mathrm{~m} \mathrm{~s}^{-1}$, los cuales superan ligeramente el intervalo de $u^{*}$ típico $\left(0,05-0,3 \mathrm{~m} \mathrm{~s}^{-1}\right)$ propuesto por Stull (1988). Los resultados obtenidos muestran un ciclo diario con valores de $u^{*}$ mínimos durante la madrugada y máximos entre el mediodía y las primeras horas de la tarde.

Los valores significativos de $u^{*}$, para los vientos del sur, obtenidos a partir de la relación entre $u^{*}$ y los valores promedios horarios positivos de $s \tau_{y}$ (Fig. 8) oscilan entre alrededor de 0 y $0,88 \mathrm{~m} \mathrm{~s}^{-1}$ (Fig. 11), con una moda en torno a $0,086 \mathrm{~m} \mathrm{~s}^{-1}$. En los datos se observó un ciclo anual con un aumento de la frecuencia de mayores velocidades de fricción en primavera y verano, y velocidades mínimas en invierno.

\subsection{Flujo horizontal}

El flujo $G$ mostró un incremento, de 2 a 3 órdenes de magnitud durante la primavera y verano, especialmente en octubre y noviembre (Fig. 12), respecto del resto de los meses de los años monitoreados $(1999,2000$ y 2001).
Al comparar distintos intervalos de $u^{*}$, que exceden un $u^{*}(D p)$ calculado de $0,24 \mathrm{~m} \mathrm{~s}^{-1}$ con los valores de $G$ determinados para el año 2000 se observó que una mayor frecuencia de velocidades de fricción altas $\left(0,4<u^{*}<0,5 \mathrm{~m} \mathrm{~s}^{-1}\right)$ en primavera coincide con el aumento de $G$.

Espacialmente, se observó una mayor cantidad de partículas capturadas en la estación DGAC (St2) que en ORICA (St1) durante todo el período de monitoreo.

\subsection{Granulometría y textura superficial de las partículas transportadas}

El material capturado en la estación Orica (St1) durante el año 2000 se caracterizó por diámetros $(D p)$ inferiores a $1.000 \mu \mathrm{m}$ con modas principales en los rangos 355-425 $\mu \mathrm{m}, 250-300 \mu \mathrm{m}, 180-212 \mu \mathrm{m}$, $125-150 \mu \mathrm{m}$ y $75-90 \mu \mathrm{m}$, siendo la más importante la observada entre $125-150 \mu \mathrm{m}$, coincidente con la moda dominante en la fracción fina de las muestras de suelo analizadas.

Asimismo, se observaron variaciones espaciales y temporales en el diámetro de las partículas (Fig. 13). Verticalmente se observó una relación inversa entre el diámetro máximo $\left(D p_{\text {max }}\right)$ y la altura (Tabla 1), mientras que superficialmente se observaron diferencias de tamaño entre los sedimentos capturados en las dos estaciones, con mayor cantidad de partículas finas en la estación DGAC (St2) (Tabla 2). Esto coincide con el mayor contenido de partículas de menor tamaño (arenas finas a muy finas) observado en los sedimentos superficiales existentes al sur de esta estación. Temporalmente se pudo apreciar que el $D p_{\max }$ transportado aumentó en primavera, particularmente durante octubre, en ambas estaciones de captura y en los tres años monitoreados.

Las partículas capturadas presentan superficies pulidas y poseen buen redondeamiento, especialmente los fragmentos de conchas y los granos de cuarzo que además poseen esfericidad moderada a alta (según Folk, 1951). Los feldespatos, en general, presentan superficies rugosas y angulosas. Algunos fragmentos líticos y cuarzos presentan irregularidades interpretadas como marcas de impacto (Fig. 14).

Las características observadas en las partículas dominantes indican que la madurez textural es moderada (según Folk, 1951). 


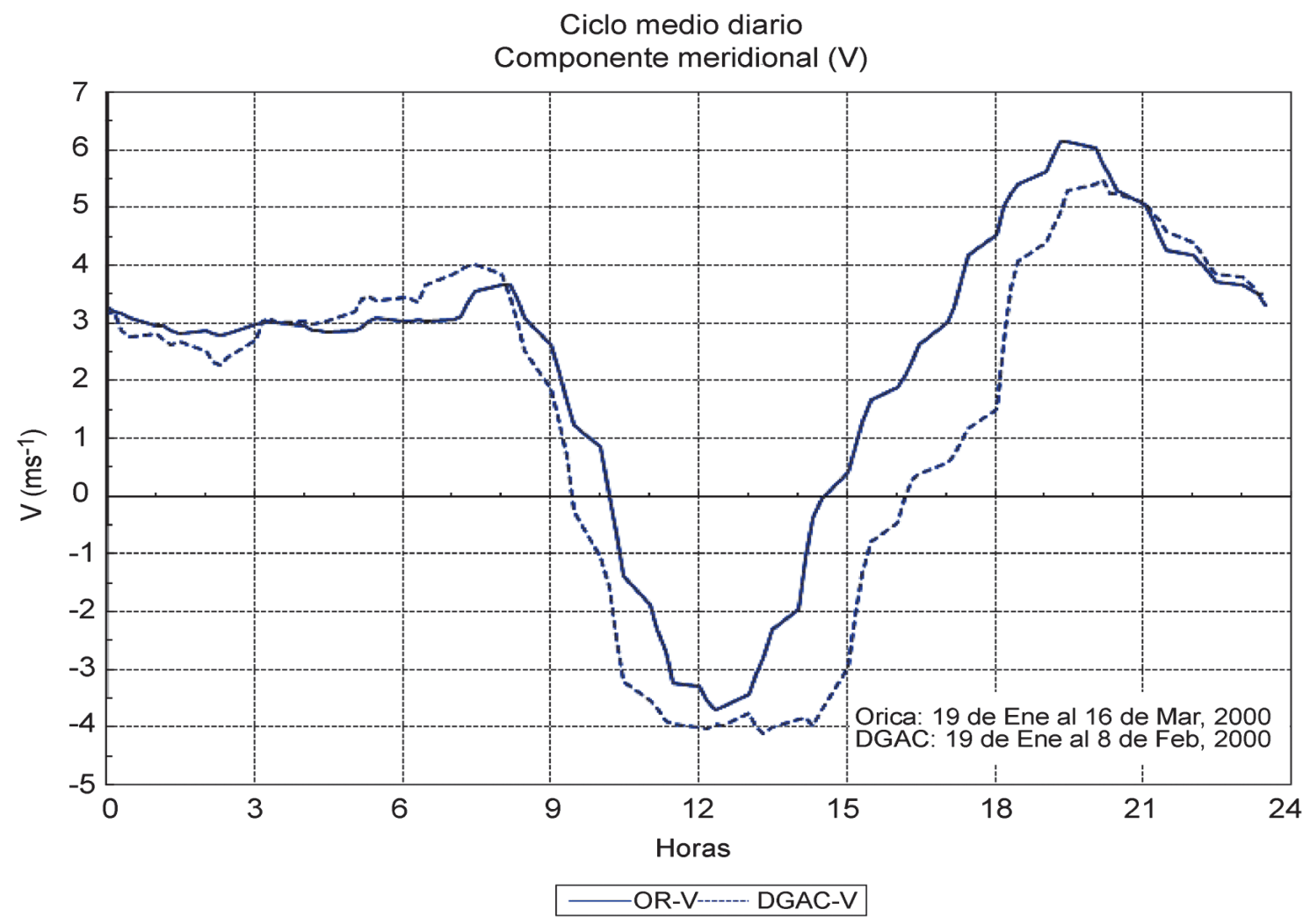

FIG. 9. Ciclo medio diario para la magnitud de la componente meridional del viento (v) durante el experimento EOLOS-2000 en las estaciones Orica (St1) y DGAC (St2).

TABLA 1. VARIACIÓN TEMPORAL (MES) Y ESPACIAL (ALTURA $Z$ ) DEL DIÁMETRO MÁXIMO $\left(D P_{M U X}\right)$ DE LAS PARTÍCULAS TRANSPORTADAS EÓLICAMENTE $(D p \leq 1.000 \mu \mathrm{m})$ DURANTE EL AÑO 2000 EN LA ESTACIÓN ORICA (St1).

\begin{tabular}{crcc}
\hline & \multicolumn{3}{c}{ Dp máx $(\boldsymbol{\mu m})$} \\
\cline { 2 - 4 } Período & $\mathbf{z}=\mathbf{1 0} \mathbf{c m}$ & $\mathbf{z}=\mathbf{5 1} \mathbf{c m}$ & $\mathbf{z}=\mathbf{1 1 2} \mathbf{c m}$ \\
\hline Ene & 1.180 & 850 & 850 \\
Feb & 1.000 & 710 & 600 \\
Mar & 1.180 & 850 & 710 \\
Abr & 1.180 & 710 & 710 \\
May & 1.180 & 710 & 710 \\
Jun & 1.180 & 1.000 & 850 \\
Jul & 850 & 710 & 1.000 \\
Ago & 1.000 & 850 & 710 \\
Sep & 710 & 710 & 425 \\
Oct & 2.000 & 1.180 & 1.180 \\
Nov & 1.400 & 1.000 & 850 \\
Dic & 1.400 & 710 & 850 \\
\hline
\end{tabular}

\subsection{Mineralogía de las partículas transportadas}

$\mathrm{Al}$ analizar la composición mineralógica a la lupa binocular por intervalo de tamaño del sedimento capturado se observó que ésta es homogénea independientemente del diámetro de las partículas. Los principales componentes son cuarzo y feldespatos $(65-85 \%)$, los que fueron considerados conjuntamente debido a que por poseer ambos densidades similares $\left(\rho_{\text {cuarzo }}=2.65 \mathrm{gm} \mathrm{cm}^{3}, \rho_{\text {feldespato }}=2.55-2.75\right.$ $\mathrm{gm} \mathrm{cm}^{3}$ ) (Dana, 1951) son igualmente susceptibles de ser transportados. Otros componentes del material capturado son fragmentos líticos principalmente de origen volcánico (10-15\%), restos de conchas (3-15\%), agregados de sales $(\sim 3 \%)$ y cristales individuales de micas y anfíbolas ( $<1 \%)$ (Fig. 14).

El predominio de cuarzo sobre feldespato observado en forma cualitativa en el análisis de lupa binocular indica que la madurez composicional de los depósitos es moderada (según Folk, 1951). 


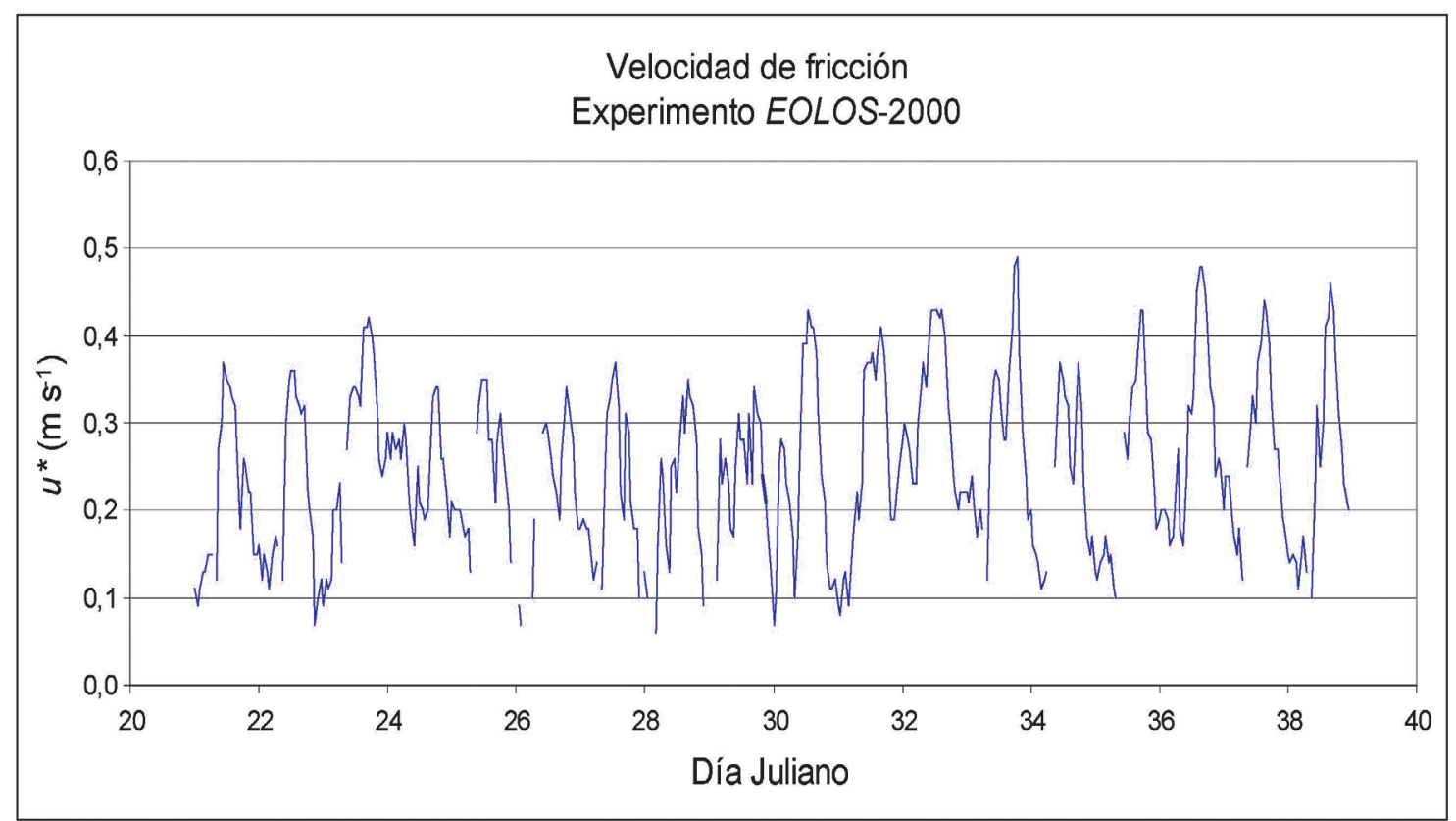

FIG. 10. Valores de $u^{*}$ obtenidos a partir de la medición de perfiles de viento y las ecuaciones de Businger-Dyer (ver texto).

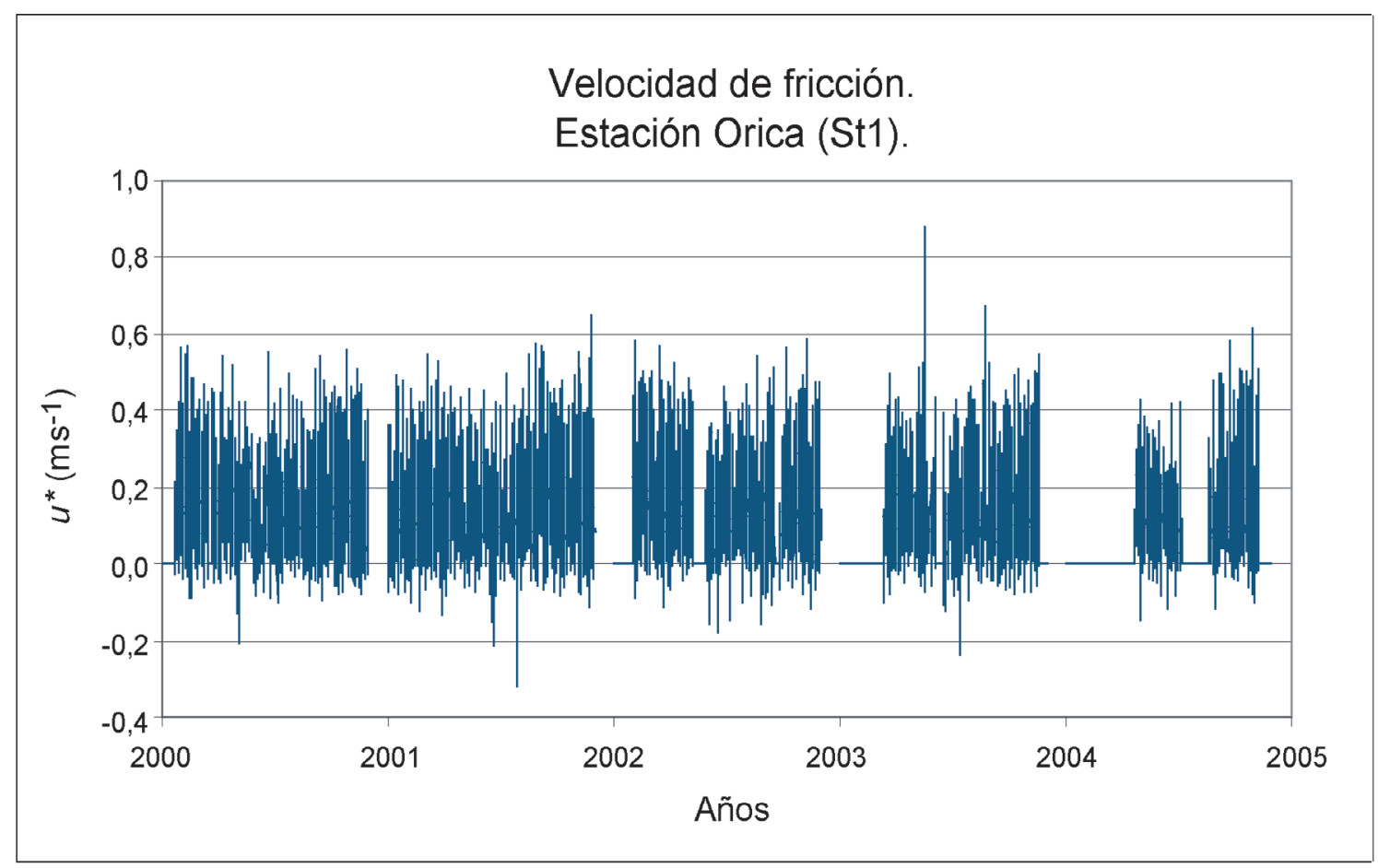

FIG. 11. Valores medio horarios (obtenidos de registros cada 15 minutos) de $u^{*}$ para el período 2000-2005 generados a partir de la relación entre $u^{*}$ durante el experimento EOLOS-2000 y la componente meridional del seudoesfuerzo superficial del viento $\left(s \tau_{y}\right)$ de la estación meteorológica permanente. Períodos en blanco indican ausencia de datos. 
TABLA 2. COMPARACIÓN ENTRE LOS PORCENTAJES RELATIVOS DE LOS TAMAÑOS PRINCIPALES DE LAS PARTÍCULAS TRANSPORTADAS $(D p \leq 1.000 \mu \mathrm{m})$ EN LAS ESTACIONES ORICA (St1) Y DGAC (St2) DURANTE JUNIO, SEPTIEMBRE Y OCTUBRE DE 2000.

\begin{tabular}{|c|c|c|c|c|c|c|c|}
\hline \multirow{3}{*}{\multicolumn{2}{|c|}{ Altura trampa (cm) }} & \multicolumn{6}{|c|}{ St1 (ORICA) } \\
\hline & & \multicolumn{6}{|c|}{ \% TAMAÑOS } \\
\hline & & $138 \mu \mathrm{m}$ & $116 \mu \mathrm{m}$ & $83 \mu \mathrm{m}$ & $69 \mu \mathrm{m}$ & $58 \mu \mathrm{m}$ & $42 \mu \mathrm{m}$ \\
\hline $\mathrm{J}$ & 10 & 13 & 7 & 2 & 1 & 1 & 0 \\
\hline $\mathrm{U}$ & 51 & 3 & 3 & 3 & 3 & 1 & 1 \\
\hline $\mathrm{N}$ & 112 & 7 & 6 & 6 & 8 & 9 & 9 \\
\hline $\mathrm{S}$ & 10 & 25 & 15 & 5 & 3 & 3 & 1 \\
\hline E & 51 & 4 & 4 & 11 & 10 & 12 & 12 \\
\hline $\mathrm{P}$ & 112 & 8 & 8 & 18 & 2 & 11 & 12 \\
\hline $\mathrm{O}$ & 10 & 14 & 4 & 2 & 2 & 1 & 0 \\
\hline $\mathrm{C}$ & 51 & 1 & 1 & 1 & 2 & 2 & 1 \\
\hline $\mathrm{T}$ & 112 & 0 & 4 & 10 & 12 & 7 & 11 \\
\hline
\end{tabular}

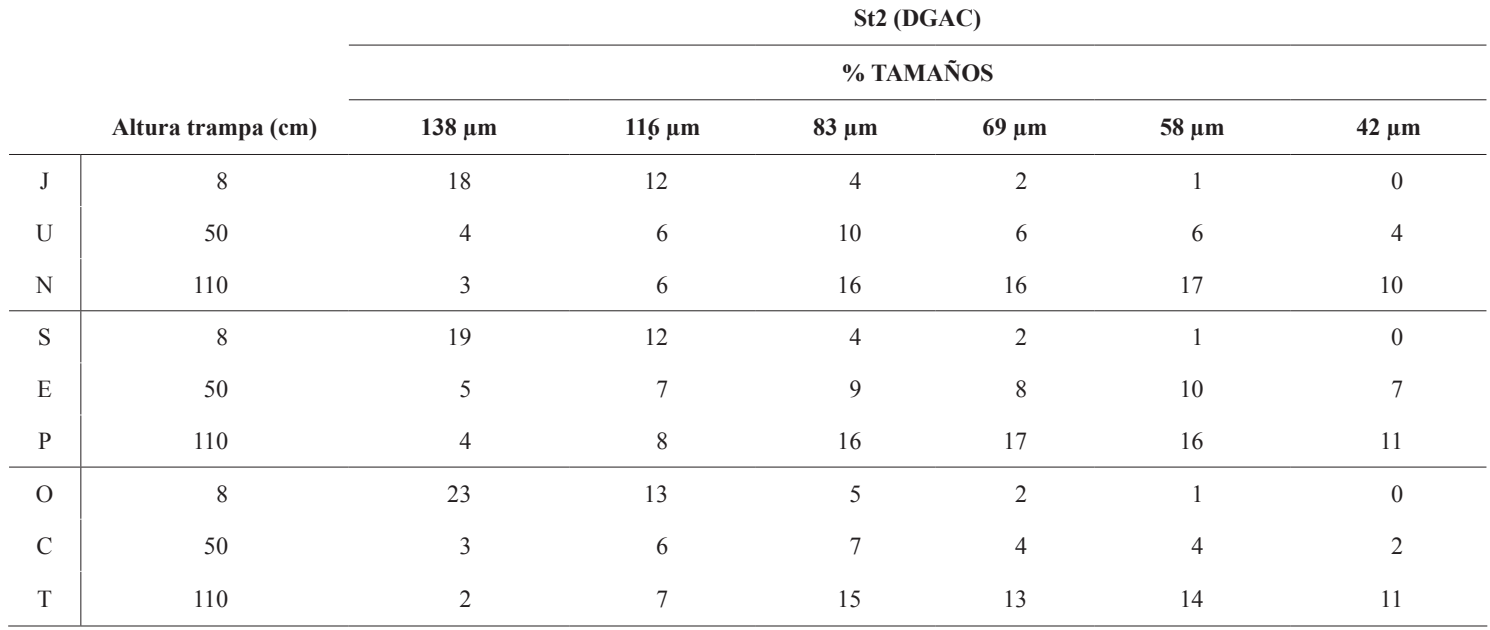

\subsection{Características granulométricas y textura- les de los sedimentos de la pampa}

La granulometría de los sedimentos de la Pampa Mejillones varía desde gravas gruesas a arenas muy finas, con un patrón bimodal o polimodal en la mayoría de los casos (Fig. 15). Las curvas de distribución granulométrica total muestran un predominio de los sedimentos gruesos $(>1.000 \mu \mathrm{m})$, excepto para las muestras SM St1, SM St2, SM12 y SM14 donde la frecuencia de los dos intervalos de tamaños más importantes, uno asociado a sedimentos gruesos $(\geq 4.750 \mu \mathrm{m}$ para SM St1, SM St2 y SM12, y 1400 para SM14) y otro a finos ( $125 \mu \mathrm{m}$ en todos los casos), es similar. Las partículas gruesas (grava fina a grava gruesa) presentes en las muestras se asocian a las crestas de los paleocordones litorales regresivos alzados; mientras que los depósitos finos se relacionan con arenas eólicas depositadas en las artesas formadas entre los cordones.

La fracción de partículas eólicamente transportables indica que la mayoría de las muestras poseen modas comunes en los rangos 1.000-1.180 $\mu \mathrm{m}, 500-600$ $\mu \mathrm{m}, 355-425 \mu \mathrm{m}, 250-300 \mu \mathrm{m}, 180-212 \mu \mathrm{m}, 125-150$ $\mu \mathrm{m}$ y $75-90 \mu \mathrm{m}$. Considerando el porcentaje relativo entre las modas más frecuentes (125-150 $\mu \mathrm{m}$ y 1.000- 

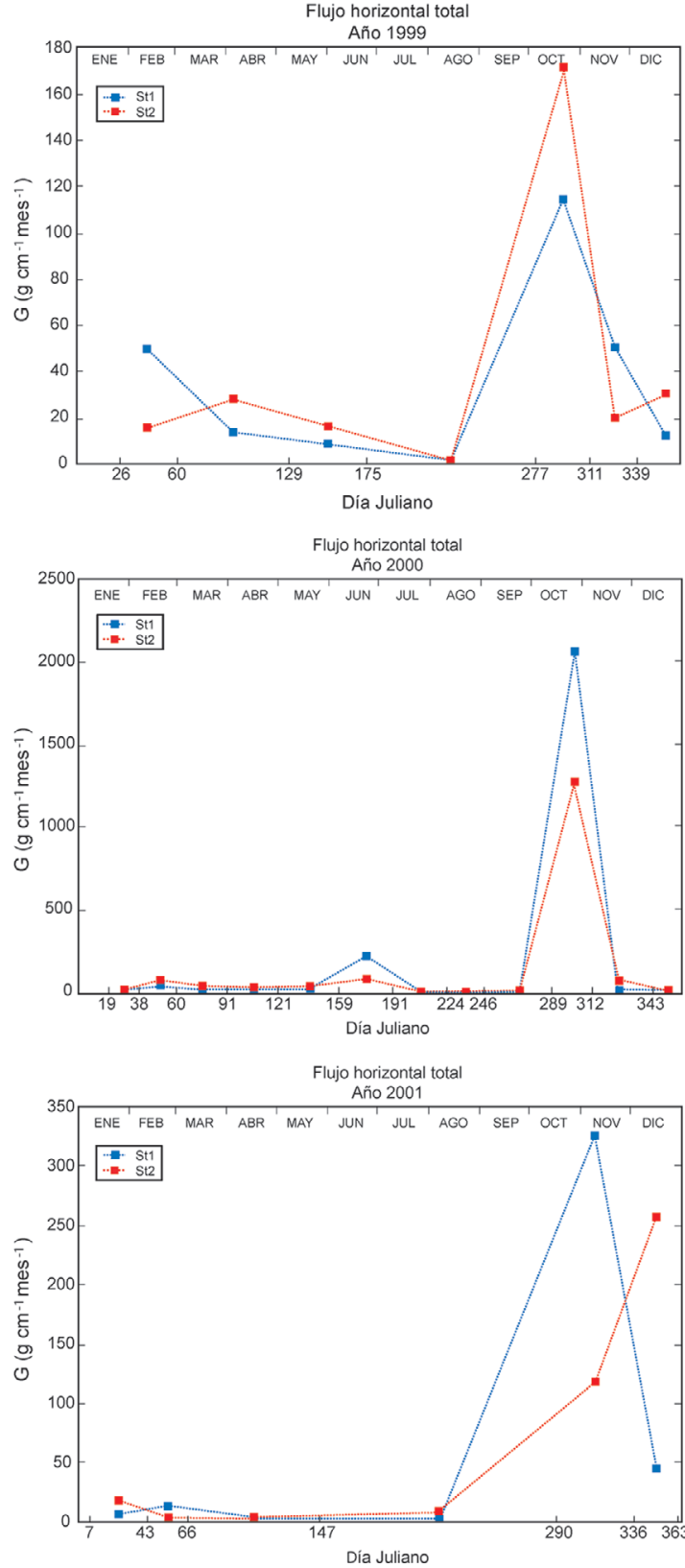

FIG. 12. Magnitud del flujo horizontal de partículas $(G)$ para los años 1999, 2000 y 2001 en las estaciones Orica (St1) y DGAC (St2). El punto con el correspondiente valor de $G$ se ubica en el centro de cada período de muestreo.

$1.180 \mu \mathrm{m})$ se reconocen 2 poblaciones principales, una en la que predominan las partículas gruesas (arena media a arena gruesa) (SM08 y SM15) y otra donde se destacan las partículas finas (arena muy fina a arena fina) (SM St1, SM St2, SM12, SM13,
SM14, SM16 y SM18). La granulometría de las mismas muestra una tendencia a la disminución en el tamaño de grano de oeste a este y de sur a norte a lo largo de la pampa.

\subsection{Mineralogía de los sedimentos de la pampa}

El análisis mineralógico semicuantitativo por difracción de rayos $\mathrm{X}$ de los sedimentos de la Pampa Mejillones evidenció una composición similar a la del material capturado en las trampas de partículas de la estación Orica (St1), dominada por cuarzo $(51 \%)$ y albita (38\%) (Fig. 16). Observaciones al microscopio electrónico de barrido indicaron texturas superficiales de las partículas como la presencia de dos tipos de superficies: una antigua con bordes redondeados y una más nueva definida por bordes agudos (Fig. 17). Además, en la superficie de los granos se observaron trazas de impacto típicamente eólicas, reconocidas también en los análisis al microscopio estereográfico, y texturas de disolución asociadas a fenómenos de pedogénesis en la pampa (Fig. 17).

\section{Discusión}

\subsection{Aspectos sedimentológicos de las partículas transportadas}

La granulometría del material capturado en las trampas, de tamaños inferiores a $1.000 \mu \mathrm{m}$, refleja que los rangos de tamaños dominantes $(75-90 \mu \mathrm{m}$ y $125-150 \mu \mathrm{m})$ son asociables a las modas más importantes de la fracción fina $(<250 \mu \mathrm{m})$ de los sedimentos de la pampa. Las partículas capturadas presentan variaciones espaciales, con una disminución del $D p_{\text {máx }}$ con la altura, concordando con Goossens (1985) quien observó una relación inversa entre el tamaño medio de las partículas y la altura. Sin embargo, los resultados obtenidos por Breuning-Madsen y Awadzi (2005) a partir de un experimento realizado en Ghana, Africa occidental, muestran una situación opuesta, probablemente a causa de distintas condiciones aerodinámicas alrededor de los captores, mayor distancia a la fuente de polvo y, por lo tanto, un transporte dominado por la decantación de partículas movilizadas en suspensión, evidenciando que estos factores son aspectos de importancia por considerar en el análisis de las partículas transportadas en estudios de este tipo. Además, se observan diferencias entre ambas esta- 

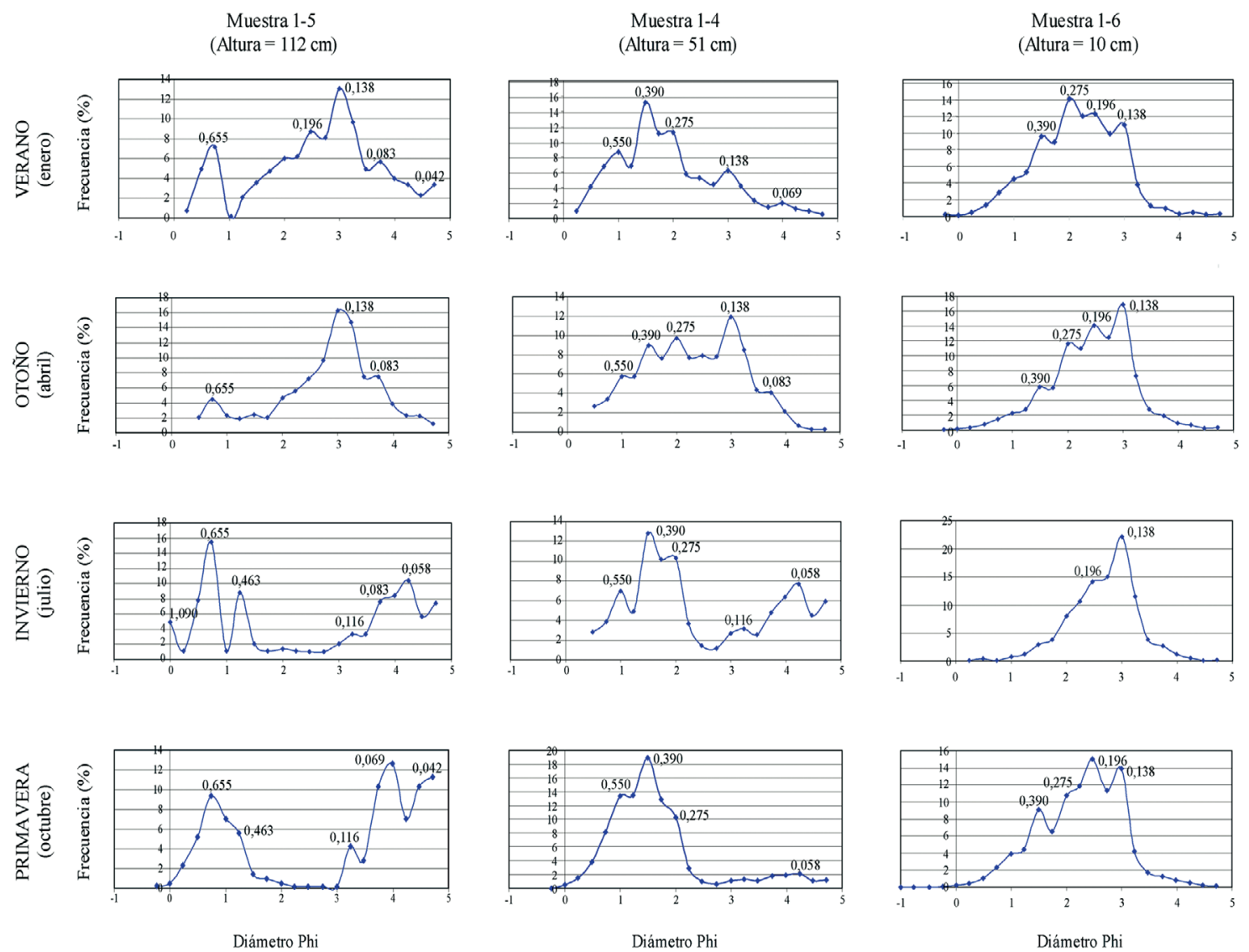

FIG. 13. Variación de la granulometría del material capturado en la estación Orica (St1) según la altura de la trampa y el mes del año. Se destacan los intervalos de tamaños principales en cada caso.

ciones de captura con una mayor concentración de partículas finas en la estación más cercana a la costa DGAC (St2) asociadas a diferencias en la sedimentología de los sedimentos presentes al sur de ésta (Tabla 2), debido a la selección producida por efecto del viento del sur-suroeste durante el transporte de partículas hacia el norte y el efecto topográfico que ejerce el morro Mejillones, ubicado en el sector occidental de la península (Fig. 1), el cual actuaría como barrera formando un corredor que ayudaría a canalizar el viento en dirección al norte.

El incremento del $D p_{\operatorname{mix}}$ transportado, con tamaños en general entre 425 y $1.000 \mu \mathrm{m}$ durante primavera, especialmente en octubre, donde sin embargo, para el año 2000 se observó una partícula de diámetro de $2.000 \mu \mathrm{m}$, coincide con el período de mayor transporte.

La mineralogía del material transportado resultó ser independiente del diámetro de los granos, para partículas inferiores a $425 \mu \mathrm{m}$, el tamaño más grueso analizado. Esto se explica por el predominio de partículas livianas (cuarzo y feldespato) en las fracciones eólicamente transportables del suelo.

Las características texturales de las partículas de cuarzo y fragmentos de conchas como superficies pulidas, bordes redondeados y marcas de impacto en ' $\mathrm{V}$ ' evidencian la importancia del transporte eólico de acuerdo a lo señalado por Barbey et al. (1974). Por su parte, los feldespatos presentan en general superficies rugosas y angulosas. Esto indica la existencia de dos poblaciones con distinta madurez textural.

Las observaciones realizadas al microscopio de barrido muestran que algunos de los granos transportados tienen una historia compleja indicada por las presencia de trazas de impacto y la sobreposición de superficies más recientes con bordes agudos, a superficies antiguas con bordes redondeados. Esta 

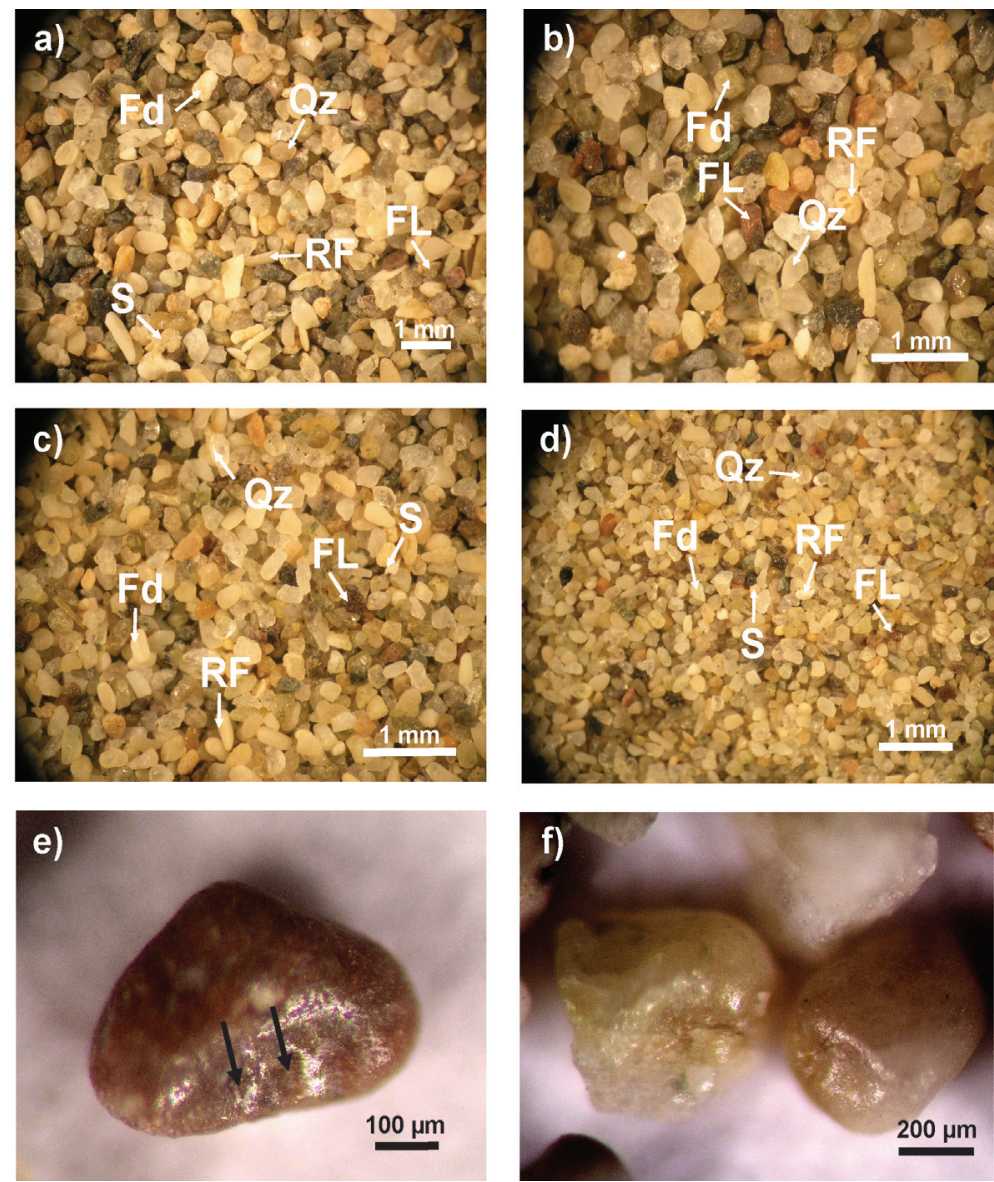

FIG. 14. Mineralogía principal y textura superficial de las partículas capturadas por la trampa inferior $(10 \mathrm{~cm})$ durante octubre de 2000 en la estación Orica (St1). a. $D p=355-425 \mu \mathrm{m}$; b. $D p=250-300 \mu \mathrm{m} ;$ c. $D p=180$ $212 \mu \mathrm{m} ;$ d. $D p=125-150 \mu \mathrm{m}$; e. Marcas de impacto en ' $V$ ' en granos de cuarzo; f. Superficies pulidas en granos de cuarzo (Fd: feldespatos; Qz: cuarzo; RF: restos fósiles; FL: fragmentos líticos; S: sales). última característica se asocia probablemente al origen de los granos desde antiguas líneas de playa, actualmente emergidas (Higgs, 1979). Las texturas de disolución observadas en las partículas son interpretadas como el resultado de una estadía larga sin disturbios, pero afectada por procesos pedogenéticos durante la permanencia de los granos en el suelo de la pampa (Le Roux et al., 2008).

\subsection{Parametrización y estacionalidad del trans- porte eólico}

El valor promedio de $\mathrm{z}_{0}$ obtenido $\left(\sim 10^{-3} \mathrm{~m}\right) \mathrm{du}-$ rante el experimento de 18 días, en enero-febrero de 2000, concuerda con lo esperado para planicies desérticas según lo indicado en la literatura (Stull, 1988).

Los valores horarios de $u^{*}$ determinados a partir de los perfiles de viento muestran un ciclo diario con valores más altos durante el mediodía y en las primeras horas de la tarde. Este ciclo diario está condicionado por los vientos regionales que responden al gradiente térmico tierra-mar que alcanza su máximo en la tarde con cielos despejados. A escala anual se observa un aumento de los valores de $u^{*}$ en primavera y verano, con los valores más bajos en invierno. Este comportamiento es consistente con la influencia del anticiclón subtropical y la superposición del efecto del gradiente termal regional tierra-mar que se incrementa particularmente durante el período primaveral-estival a lo largo de la costa (Rutllant et al., 1998; Strub et al., 1998).

El incremento en el flujo horizontal $G$ durante la primavera se caracterizó por un desfase de algunas semanas entre el inicio de la ocurrencia de velocidades altas $\left(u^{*}>0,4 \mathrm{~m} \mathrm{~s}^{-1}\right)$, y el máximo transporte horizontal de partículas (Fig. 18). Estos resultados difieren en parte de lo indicado por algunos autores (e.g., Bagnold, 1941; Marticorena y Bergametti, 1995; 


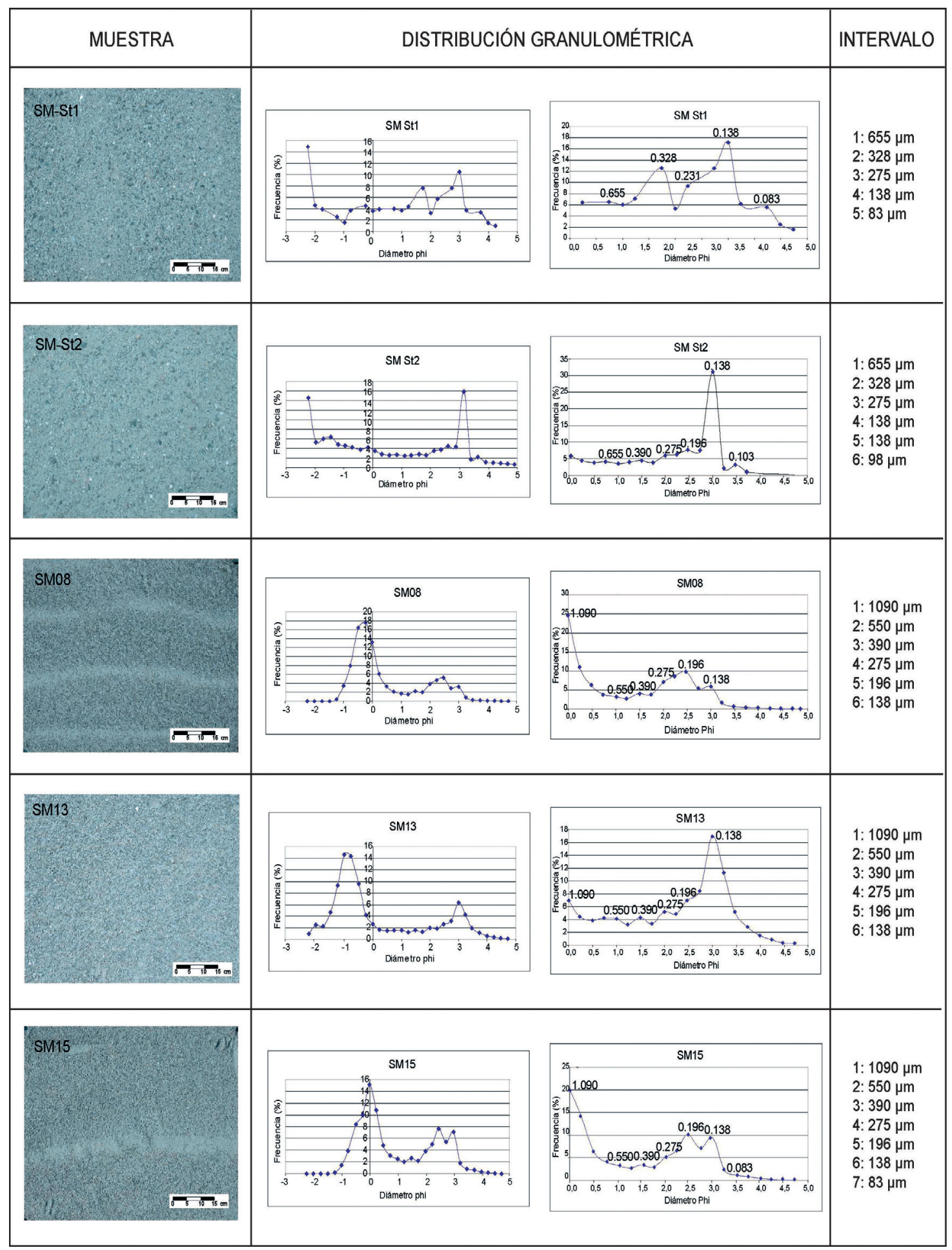

FIG. 15. Fotografías, curvas de distribución de tamaños de partículas totales y partículas eólicamente transportables $(\leq 1.000 \mu \mathrm{m}$, recalculadas al 100\%) correspondientes a las muestras de sedimento recolectadas en la Pampa Mejillones. Se destacan los intervalos de tamaños principales. 

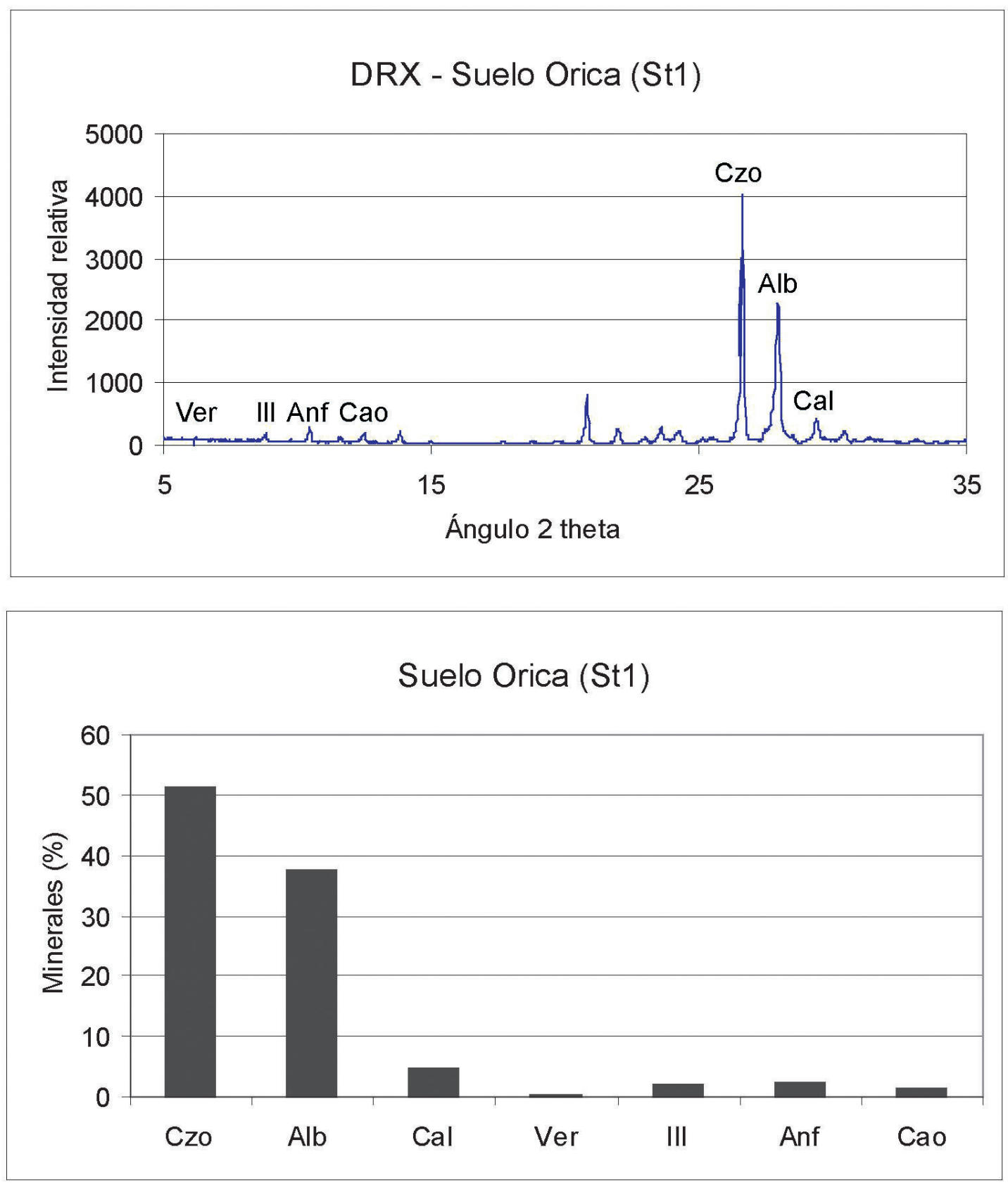

FIG. 16. Arriba: Composición mineralógica por análisis de difracción de rayos X para una muestra de suelo de la estación Orica (St1). Abajo: Porcentaje relativo de los minerales en la muestra (Czo: cuarzo; Alb: albita; Cal: calcita; Ver: vermiculita; Ill: illita; Anf: anfíbola; Cao: caolinita).

Alfaro, 1997) que señalan una simultaneidad entre ambos procesos. Este desfase observado en la Pampa Mejillones se interpreta como una preparación de los sedimentos superficiales, durante los períodos de velocidades de fricción relativamente altas al inicio de la primavera, consistente en una remoción de las partículas gruesas en la superficie del suelo seco de la pampa, permitiendo el transporte de las partículas más finas durante los episodios de velocidades de fricción máximas. A esto podría también contribuir 

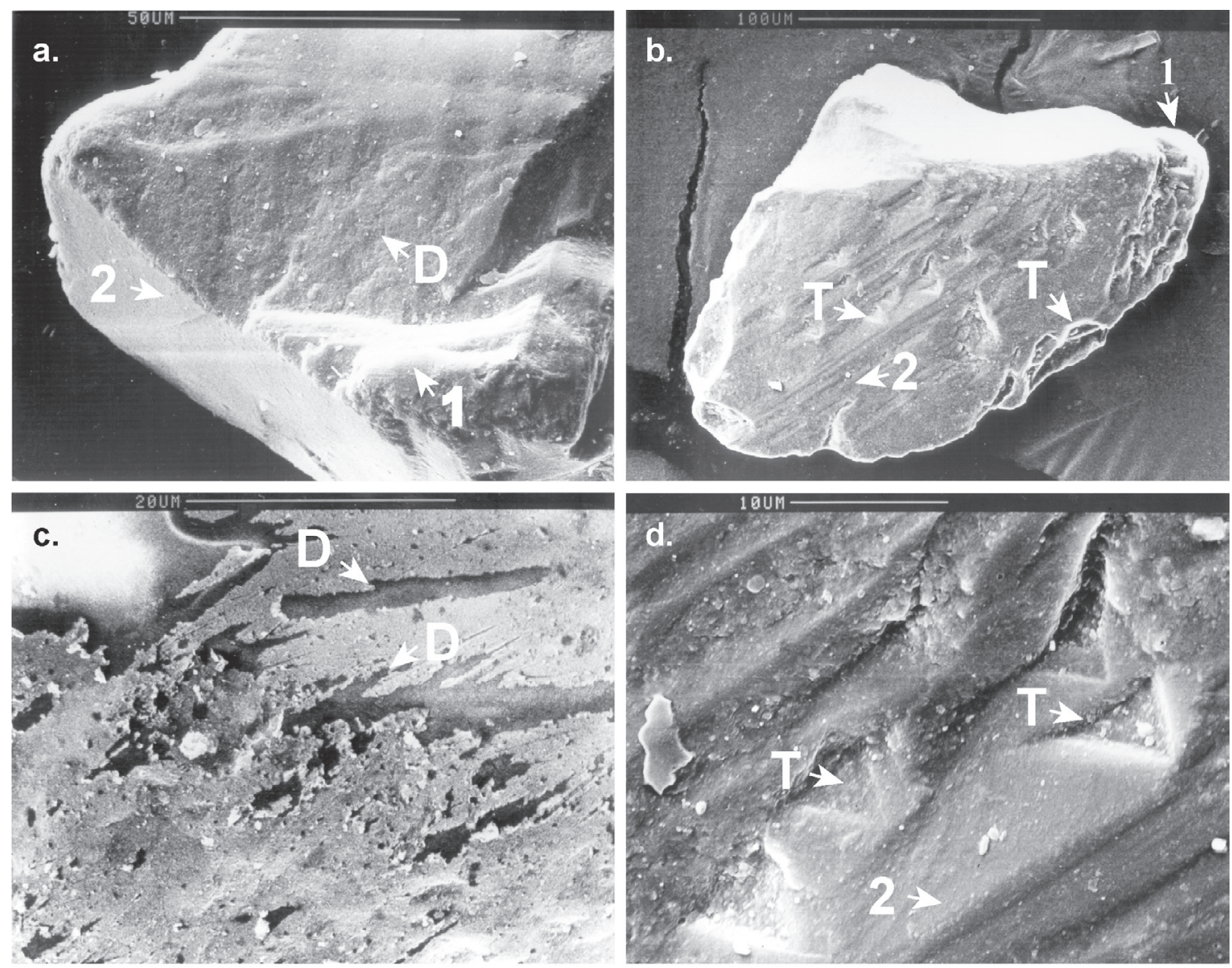

FIG. 17. Características texturales de granos de cuarzo de los sedimentos de la Pampa Mejillones. Fotografías de análisis al microscopio electrónico de barrido de granos de cuarzo. T: marcas de impacto en 'V'; D: estructuras de disolución; 1: superficie antigua con bordes redondeados; 2: superficie más reciente con bordes angulosos.

la presencia de remolinos de polvo que se observan particularmente en condiciones de velocidades de viento bajas y alta insolación.

\subsection{Transporte eólico y clima del desierto costero}

Al correlacionar linealmente los valores de $s \tau_{y}$ en Orica (St1), promediados entre las 12:00 y las 24:00 hrs (intervalo de mayor intensidad del viento sur), con los valores correspondientes de las estaciones Caleta Constitución $\left(\mathrm{R}^{2}=0,56\right)$ y Cerro Moreno $\left(\mathrm{R}^{2}=0,41\right)$ (Fig. 1) se pone en evidencia que los mismos vientos responsables del arrastre eólico de partículas en la Pampa Mejillones son los que producen la surgencia costera en la península de Mejillones. Una comprobación climatológica de este resultado deberá hacerse considerando un período de tiempo mayor para las correlaciones.

Los mayores valores de $s \tau_{y}$ encontrados en Cerro Moreno relativos a los de Orica (St1) y DGAC (St2) (Fig. 19) se deben a que en Cerro Moreno la brisa marina diurna se superpone con los vientos regionales del sur aumentando la magnitud de $s \tau_{y}$ mientras que en Orica (St1) y DGAC (St2) se produce la situación opuesta; la brisa marina en la bahía de Mejillones es del norte, generando valores negativos de $s \tau_{y}$ (hacia el sur) entre aproximadamente media mañana y media tarde, momento a partir del cual los vientos regionales del sur dominan sobre la brisa local. Esto se confirma con el mayor retardo en la aparición de los vientos del sur en DGAC (St2). Cabe señalar 
Orica (St1), año 2000.
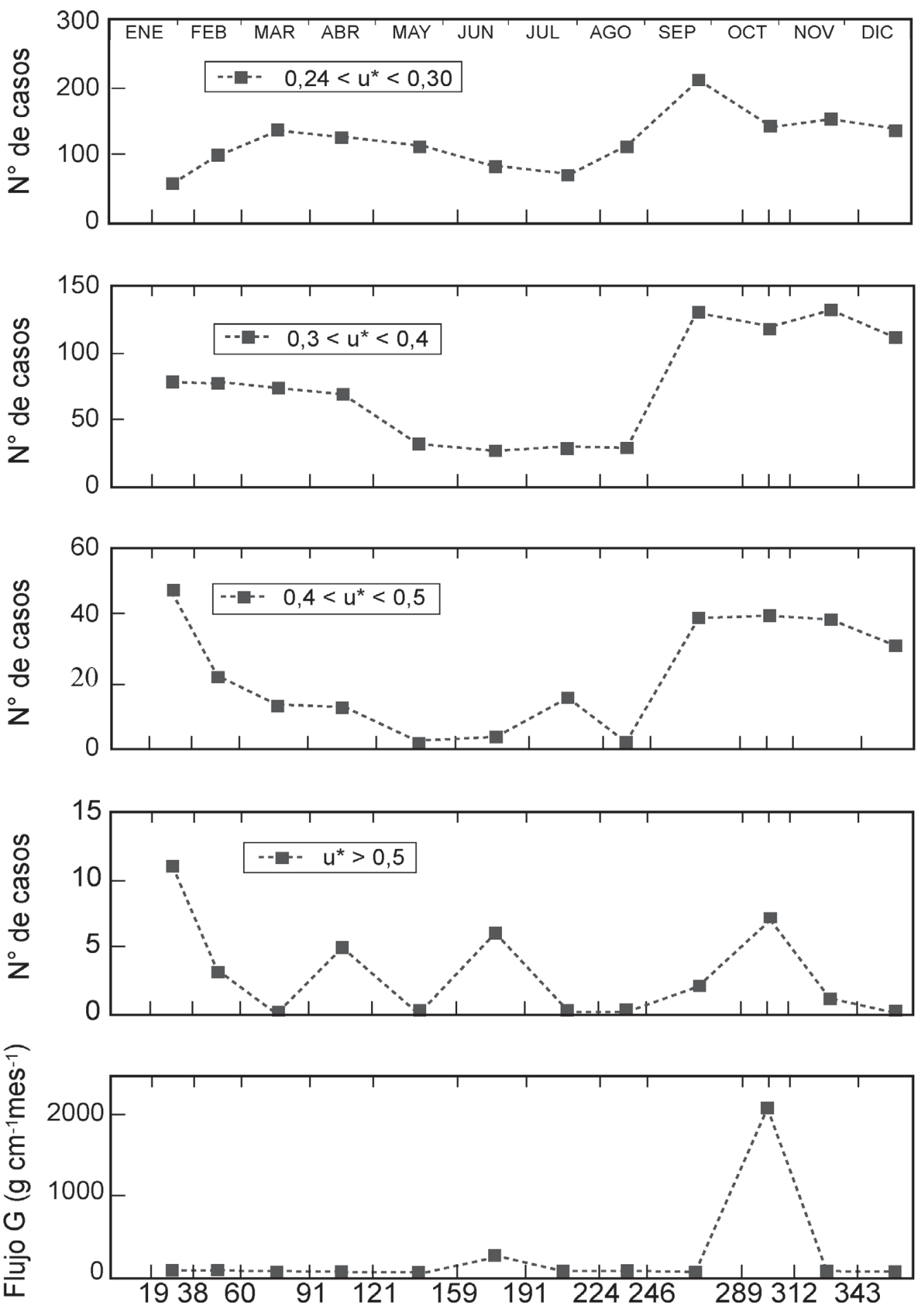

Día Juliano

FIG. 18. Variabilidad temporal del número de casos en que $u *$ pertenece a cierto intervalo de valores. En el cuadro inferior se muestra la magnitud del flujo $G$ para los mismos períodos. Los datos corresponden a la estación Orica (St1) durante el año 2000. 


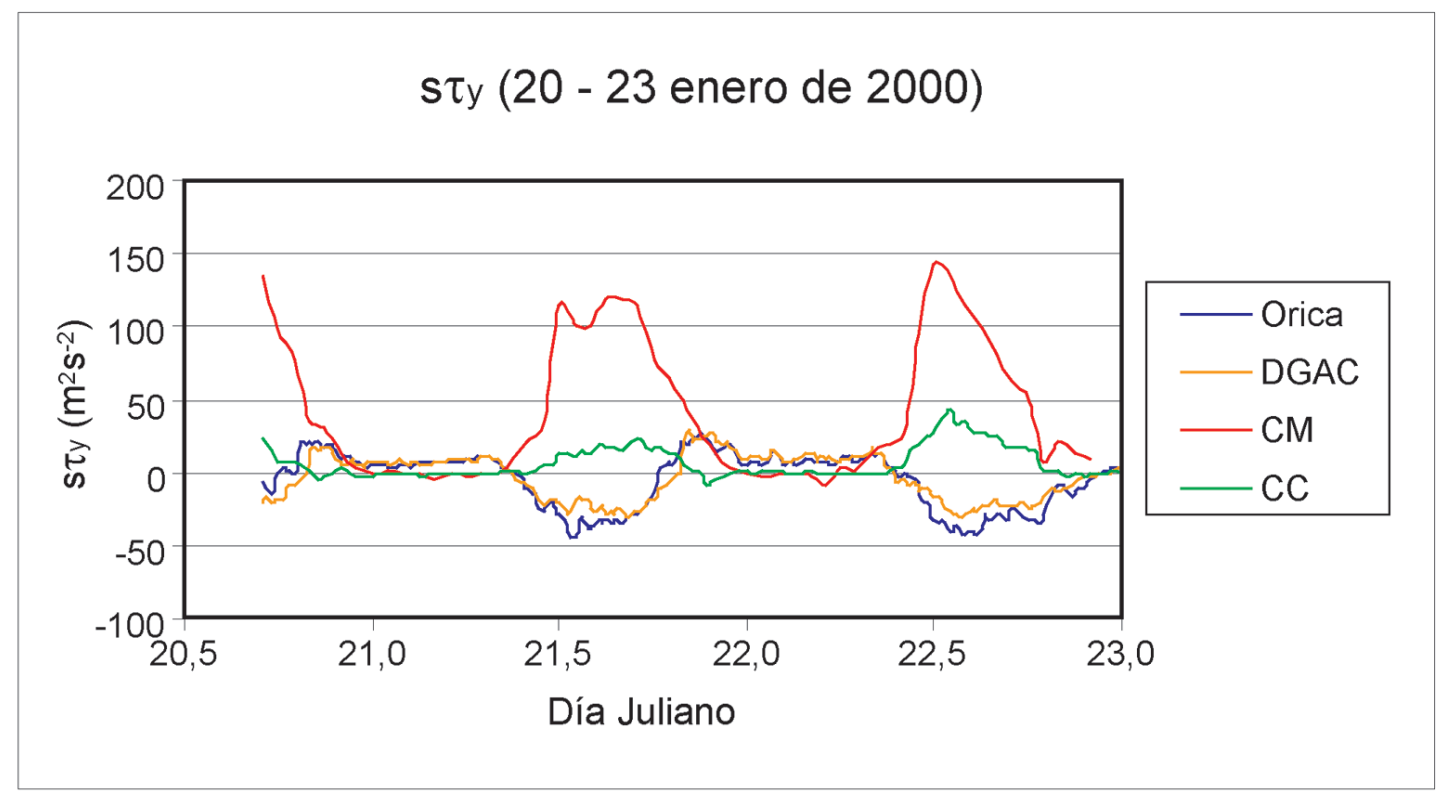

FIG. 19. Comparación de los valores promedios de la componente meridional del seudoesfuerzo superficial del viento $\left(s \tau_{y}\right)$ para las distintas estaciones meteorológicas durante los días 20,21, 22 y 23 de enero de 2000. La frecuencia de promedios disponibles es cada 15 minutos para las estaciones Orica (St1) y DGAC (St2), cada 30 minutos para Caleta Constitución (CC) y horarios (puntuales) para Cerro Moreno (CM).

que la velocidad del viento en Cerro Moreno se mide a $10 \mathrm{~m}$ de altura, exagerando las magnitudes del viento sur en esta estación al compararlas con las otras estaciones. Por su parte, la estación Caleta Constitución, ubicada en el borde occidental de la península, no recibe influencia apreciable de las brisas locales, representando así condiciones regionales forzantes de la surgencia costera.

En consecuencia, un aumento del flujo horizontal de partículas incrementaría el aporte de granos líticos al fondo de la bahía, conjuntamente con un incremento en el aporte de sedimentos orgánicos debidos al aumento de la biomasa planctónica y productividad primaria asociados a los procesos de surgencia (Vargas et al., 2004). El estudio de estos componentes (partículas líticas y restos pelágicos) presentes en los sedimentos laminados de la bahía Mejillones ofrece un gran potencial para reconstituciones de alta resolución (escala decenal y secular) de la variabilidad de la intensidad del viento sur-suroeste dominante en la zona y de los eventos de surgencia costera, basado en reconstrucciones paleooceanográficas a partir del estudio de testigos de fondo marino extraídos de la bahía de Mejillones (Vargas et al., 2004, 2007).

\section{Conclusiones}

Los procesos de transporte eólico en la Pampa Mejillones, dada la continua movilización de partículas, son un factor importante en la geomorfología del área. Estos procesos condicionan el desarrollo de estructuras sedimentarias, como óndulas y megaóndulas, con variaciones locales atribuibles a la topografía, como la presencia de los paleocordones y depresiones de origen tectónico, y a la dirección de los vientos dominantes.

El flujo horizontal de partículas $G$ calculado para el año 2000 creció desde valores inferiores a $10^{1} \mathrm{~g} \mathrm{~cm}^{-1}$ durante gran parte del año a valores dos a tres órdenes de magnitud superiores durante la primavera-verano. Para los años 1999 y 2001 el $G_{\text {máx }}$ calculado resultó ser un orden de magnitud menor que del año 2000, probablemente debido a la menor ocurrencia de episodios con velocidades de fricción superiores a $0,4 \mathrm{~m} \mathrm{~s}^{-1}$ y $0,5 \mathrm{~m} \mathrm{~s}^{-1}$ en esos años. 
Los $D p$ de las partículas transportadas son inferiores a $1.000 \mu \mathrm{m}$ con una moda principal en el intervalo $125-150 \mu \mathrm{m}$, similar a lo observado en la población fina de los sedimentos de la pampa. La granulometría del material capturado presenta diferencias espaciales y temporales. Verticalmente se observa una disminución del $D p_{\text {mix }}$ con un aumento de la altura (entre 710-2.000 $\mu \mathrm{m}$ para la trampa inferior, entre $710-1.180 \mu \mathrm{m}$ para la trampa intermedia y entre $425-1.180 \mu \mathrm{m}$ para la trampa superior) y superficialmente se aprecian diferencias entre el material capturado en ambas estaciones, siendo generalmente más fino en la estación DGAC (St2) debido a la granulometría del suelo al sur de ésta. Temporalmente, se observa que las partículas de mayor tamaño $\left(425 \mu \mathrm{m}<D p_{\max }<1.000 \mu \mathrm{m}\right)$ son movilizadas en octubre, correspondiente al período de mayor transporte en masa.

El valor del coeficiente de rugosidad obtenido a partir de perfiles de viento es de $\mathrm{z}_{0} \sim 10^{-3} \mathrm{~m}$, conforme a lo esperado según la teoría (Stull, 1988). Los valores de la velocidad de fricción $\left(u^{*}\right)$ muestran un ciclo diario, con velocidades máximas en la tarde y mínimas durante la noche y madrugada, y un ciclo anual, que presenta velocidades máximas en primavera-verano, y mínimas en invierno debido a la influencia permanente del anticiclón del Pacífico suroriental y el efecto del incremento diurno del gradiente termal tierra-mar durante el período primaveral-estival (Rutllant et al., 1998; Strub et al., 1998).

La variación estacional del flujo horizontal $G$ es similar a la de la velocidad de fricción, donde el máximo transporte de partículas coincide con las velocidades de fricción más altas. Sin embargo, se observa que los máximos de $u^{*}$ preceden por varias semanas a los máximos $G$. La razón de esta diferencia aún no está resuelta, pero se supone una preparación del suelo durante los inicios de la primavera cuando las condiciones del suelo y los altos valores de $u^{*}$ favorecen la remoción de las partículas gruesas, permitiendo el transporte posterior de las partículas más finas durante los eventos de fuertes vientos de la primavera.

La correlación de los vientos en las estaciones meteorológicas de la península de Mejillones sugiere una relación positiva entre la intensificación estacional del transporte de partículas y los vientos favorables a la surgencia medidos en Caleta Constitución.

\section{Agradecimientos}

Este trabajo fue financiado por los proyectos DI (I04/05-2), Fondecyt 11060484 y la Facultad de Ciencias Físicas y Matemáticas de la Universidad de Chile.

El Programa de Desarrollo en Dinámica de la Atmósfera y el Clima (PRODAC: Departamento de Investigación, Universidad de Chile) apoyó el mantenimiento de la estación meteorológica de Caleta Constitución y el trabajo de terreno. El proyecto JEAI (IRD) apoyó el mantenimiento de la estación meteorológica y muestreo de trampas de polvo en la estación Orica (St1). El proyecto ECOS-CONICYT C05U03, permitió el apoyo en terreno de S. Alfaro y S. Caquineau del Laboratoire Inter-Universitaire des Systèmes Atmosphériques (LISA), Francia, durante una reciente campaña de adquisición de datos y verificación de las hipótesis planteadas en este trabajo.

R. Muñoz (Departamento de Geofísica, Universidad de Chile), contribuyó a la optimización de los algoritmos para los cálculos de $u^{*}$ y $q_{0}$. Z. Salinas (Departamento de Geofísica, Universidad de Chile) contribuyó al tratamiento de los datos meteorológicos. H. Fuenzalida (Departamento de Geofísica, Universidad de Chile), realizó valiosos comentarios. Agradecemos también el trabajo de los evaluadores S. Bidart, F. Colombo y J. García-Ramos, cuyas observaciones nos ayudaron a mejorar considerablemente el manuscrito.

\section{Referencias}

Adetunji, M.T.; Martins, O.; Arowolo, T.A. 2001. Seasonal variation in atmospheric deposition of nitrate, sulphate, lead, zinc, and copper in southwestern Nigeria. Communications in Soil Science and Plant Analysis 32: 65-73.

Alfaro, S.C. 1997. Simulation Experimentale et Modelisation de la production d'aerosol mineral par erosion Eolienne. Ph.D. Thesis (Unpublished), Université Paris 12 Val-De-Marne, France: 176 p.

Alfaro, S.C.; Rajot, J.L.; Nickling, W. 2004. Estimation of PM20 emissions by wind erosion: main sources of uncertainties. Geomorphology 59: 63-74.

Bagnold, R.A. 1941. The Physics of Blown Sand and Desert Dunes. Chapman and Hall: 265 p. London.

Bailey, S.I. 1906. Peruvian meteorology. Annals of the Astronomical. Observatory of Harvard College 39, Part 2: 1892-1895. Cambridge, Massachusetts.

Barbey, C.; Carbonnel, J-P.; Le Ribault, L.; Tourenq, J. 1974. Mise en évidence par exoscopie des quartz de plusieurs épisodes éoliens au cours de Quaternaire mauritanien. Comptes Rendus de l'Académie des Sciences de Paris 278: 1163-1166.

Breuning-Madsen, H.; Awadzi, T. 2005. Harmattan dust deposition and particle size in Ghana. Catena 63: 23-38.

Dana, E.S. 1951. A text-book of mineralogy, with an extended treatise on crystallography and physical mineralogy. John Wiley and Sons Inc.: 851 p. London. 
Folk, R. 1951. Stages of textural maturity in sedimentary rocks. Journal of Sedimentary Petrology 21: 127-130.

Fryrear, D.W. 1986. A field dust sampler. Journal of Soil and Water Conservation 41 (2): 117-120.

Gomes, L.; Arrué, J.L.; López, M.V.; Sterk, G.; Richard, D.; Gracia, R.; Sabre, M.; Gaudichet, A.; Frangi, P. 2003a. Wind erosion in a semi-arid agricultural area of Spain: the WELSONS project. Catena 52: 235-256.

Gomes, L.; Arrué, J.L.; López, M.V.; Sterk, G.; Richard, D.; Gracia, R.; Sabre, M.; Gaudichet, A.; Frangi, J.P. 2003b. Soil aerosol production in a semi-arid agricultural area: the WELSONS project. Catena 52: $257-271$.

Goossens, D. 1985. The granulometrical characteristics of a slowly moving dust cloud. Earth Surface Processes and Landforms 10: 353-362.

Hesse, P.; McTainsh, G. 2003. Australian dust deposits: modern processes and the Quaternary record. Quaternary Science Reviews 22 (18-19): 2007-2035.

Higgs, R. 1979. Quartz surface textures of MesozoicCenozoic sands from Labrador and Western Greenland continental margins. Journal of Sedimentary Petrology 49: 599-610.

Holliday, V. 1990. Soils and landscape evolution of eolian plains: the Southern High Plains of Texas and New Mexico. Geomorphology 3 (3-4): 489-515.

Iversen, J.D.; White, B.R. 1982. Saltation threshold on Earth, Mars and Venus. Sedimentology 29: 111-119.

Le Roux, J.P. 1997. Relationship between aerodynamic entrainment threshold and hydrodynamic settling velocity of particles. Sedimentary Geology 109: 199-205.

Le Roux, J.P.; Nielsen, S.N.; Kemnitz, H.; Henríquez, Á. 2008. A Pliocene mega-tsunami and associated features in the Ranquil Formation, southern Chile. Sedimentary Geology 203: 164-180.

Marticorena, B.; Bergametti, G. 1995. Modelling the atmospheric dust cycle. Journal of Geophysical Research 100 (8): 16415-16430.

Marticorena, B.; Bergametti, G. 1996. Two-year simulations of seasonal and interannual changes of the Saharan dust emissions. Geophysical Research Letters 23 (15): 1921-1924.

Nickling, W.G.; Gillies, J.A. 1989. Emission offine-grained particulates from desert soils. In Paleoclimatology and Paleometeorology: Modern and Past Patterns of GlobalAtmospheric Transport(Leinen, M.; Sarntheim, M.; editors). Kluwer Academic Publishing: 133-165. Dordrecht.

Nishimori, H.; Tanaka, H. 2003. Simple model for the complex dynamics of dunes. In Concepts and Modelling in Geomorphology: International Perspectives (Evans, I.S.; Dikau, R.; Tokunaga, E.; Ohmori, H.; Hirano, M.; editors). TerraPub: 87-100. Tokyo.
Ortlieb, L.; Díaz, A.; Guzmán, N. 1996. A warm interglacial episode during Oxygen Isotope Stage 11 in northern Chile. Quaternary Science Reviews 15: 857-871.

Poore, R.Z.; Pavich, M.J.; Grissino-Mayer, H.D. 2005. Record of the North American southwest monsoon from Gulf of Mexico sediment cores. Geology 33 (3): 209-212.

Pye, K. 1987. Aeolian dust and deposits. Academic Press: 334 p. Londres.

Rajot, J.L.; Alfaro, S.C.; Gomes, L.; Gaudichet, A. 2003. Soil crusting on sandy soils and its influence on wind erosion. Catena 53: 1-16.

Rutllant, J.; Fuenzalida, H.; Torres, R.; Figueroa, D. 1998. Interacción océano-atmósfera-tierra en la Región de Antofagasta (Chile, $23^{\circ} \mathrm{S}$ ): Experimento DICLIMA. Revista Chilena de Historia Natural 71: 405-427.

Rutllant, J.; Montecino, V.; 2002. Multiscale upwelling forcing cycles and biological response off northcentral Chile. Revista Chilena de Historia Natural 75: 212-231.

Rutllant, J.; Fuenzalida, H.; Aceituno, P. 2003. Climate dynamics along the arid northern coast of Chile: The 1997-1998 Dinámica del Clima de la Región de Antofagasta (DICLIMA) experiment. Journal of Geophysical Research-Atmospheres 108 (D17): 4538-4542. doi:10.1029/2002JD003357.

Stout, J.; Zobeck, T. 1996. The Wolfforth field experiment: a wind erosion study. Soil Science 161: 616-632.

Strub, T.; Mesías, J.; Montecino, V.; Rutllant, J.; Salinas, S. 1998. Coastal ocean circulation off western South America. In The Sea: Coastal Oceans (Robinson, A.R.; Brink, K.H.; editors). Wiley 11: 273-313. New York.

Stull, R.B. 1988. An Introduction to Boundary Layer Meteorology. Kluwer Academic Publishers: 666 p. Dordrecht.

Thompson, L.G.; Yao, T.; Lin, P.N. 2000. A high-resolution millennial record of the south Asian monsoon from Himalayan ice cores. Science 289 (5486): 1916-1919.

Udden, J. 1898. Mechanical composition of wind deposits. Augustana Library Publication 1: 69 p.

Vargas, G.; Ortlieb, L.; Rutllant, J. 2000. Aluviones históricos en Antofagasta, Chile, y su relación con eventos ElNiño/Oscilación del Sur. Revista Geológica de Chile 27 (2): 157-176.

Vargas, G.; Ortlieb, L.; Pichon, J.J.; Bertaux, J.; Pujos, M. 2004. Sedimentary facies and high resolution primary production inferences from laminated diatomaceous sediments off northern Chile $\left(23^{\circ} \mathrm{S}\right)$. Marine Geology 211: 79-99.

Vargas, G.; Pantoja, S.; Rutllant, J.; Lange, C.; Ortlieb, L. 2007. Enhancement of coastal upwelling and interdecadal ENSO-like variability in the PeruChile Current since late $19^{\text {th }}$ century. Geophysical 


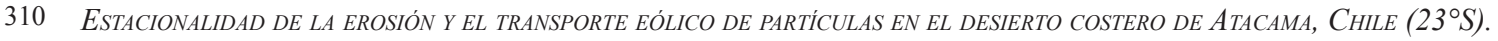

Research Letters 34. L13607. doi: 10.1029/2006 GL0288127.

Wang, X.; Zhou, Z.; Dong, Z. 2006. Control of dust emissions by geomorphic conditions, wind environments and land use in northern China: An examination based on dust storm frequency from 1960 to 2003. Geomorphology 81 (3-4): 292-308.

Wentworth, C. 1922. A scale of grade and class ter$\mathrm{ms}$ for clastic sediments. Journal of Geology 30 : 377-392.

Manuscrito recibido: agosto 02, 2007; revisado/aceptado: noviembre 19, 2008. 